\title{
Generalized theory for the dynamic analysis of thin shells with application to circular cylindrical geometries
}

\author{
by \\ Raydin Salahifar $^{\text {a }}$, Magdi Mohareb ${ }^{\text {b }}$ \\ a Baha'i Institute for Higher Education, Tehran, Iran, Tel.: +989125472292, Raydin.Salahifar@bihe.org, \\ RaydinSalahifar@yahoo.com (Corresponding author) \\ ${ }^{b}$ Department of Civil Engineering, University of Ottawa, Ottawa, ON, Canada K1N 6N5, \\ MMohareb@uottawa.ca
}

\section{Abstract}

A generalized theory is formulated for the analysis of thin shells of general curvatures based on the variational form of the Hamiltonian functional in conjunction with tensor calculus. Simplifying approximations and subtle inconsistencies made at the early stages of common classical formulations are avoided herein, and hence, the present treatment leads to field equations and boundary conditions that are accurate and consistent. The theory is then specialized to circular cylindrical shells. The well -known field equations of Flugge and Donnell-Mushtari-Vlasov (DMV) theories are recovered as consistent approximations from the present theory. Closed form solutions are then developed for the present and past cylindrical shell theories by Flugge, Timoshenko, and DMV. A comparative study is conducted to assess and quantify the effects of approximations made in classical theories on the predicted displacements and stresses.

\section{Keywords}

Thin shell theory, shells with general curvature, cylindrical shells, Hamilton principle, steady state response, Tensor-based formulation

This article is to be cited as

Salahifar R, Mohareb M, (2019) Generalized theory for the dynamic analysis of thin shells with application to circular cylindrical geometries, Thin-Walled Structures, 139: 347-36.

The copy-edited version of this article is available at: $\quad$ https://doi.org/10.1016/j.tws.2018.11.021. 
31 Thin shells are commonly encountered in structural applications, onshore and offshore pipelines, and mechanical equipment, where they are commonly subjected to a variety of static, quasi-static or dynamic loading. Under such conditions, shells may undergo a complex deformational behaviour. The literature reveals the presence of several seemingly conflicting stress-deformation theories for thin-shells. The selection for a proper shell theory in a given application requires (a) an in-depth understanding of the underlying assumptions, and (b) a critical assessment of various assumptions on the predictive ability of the theory. Within this context, the present study contributes to both objectives by developing a theory for thin shells of general geometries while keeping the assumptions and simplifications made to a minimum. The formulation is then specialized to circular cylindrical shells. By applying various approximations to the resulting formulation, other well-established cylindrical thin-walled shell theories are recovered as special cases.

\subsection{Literature Review}

Classical linear shell theories involve the work of Timoshenko [1], Novozhilov [2], Koiter [3], Flugge [4] (and [5]), Saada [6], DMV [7] and Niordson [7]. Non-linear shell theories include the work of Leonard [8], Sanders [9], Koiter [10] , [11] and Budiansky [12]. The comprehensive monograph by Leissa [13] provides a detailed report on the wide range of shell theories available. Libai and Bert [14] developed mixed variational principles for the elastic small-strains and large-rotation analysis of shells based on the Kirchhoff-Love hypothesis. Muneeb et al. [15] developed a higher order theory for the dynamic response of isotropic thermo-elastic analysis of cylindrical shells. Kolesnikov [16] formulated a refined theory for the vibration of multilayer orthotropic cylindrical shells through a series expansion of the radial displacement field in terms of the shell thickness. Through asymptotic expansion of the general equilibrium equations for a general state of stress in three-dimensional bodies, Niordson [17] formulated a two-dimensional shell theory for circular cylindrical shells. Ugrimov [18] presented a layerwise generalized theory for the elasto-dynamic analysis of multilayer plates by expanding the displacement components of each layer as power series of the transverse coordinate. Ciarlet and Gratie [19] developed an approach to minimize the quadratic problem arising in Koiter's linear shell theory. Using the Cosserat surface model,

60 Birsan [20] presented a theory for porous elastic shells by employing the Nunziato-Cowin 
theory of elastic materials with voids, to characterize the porosity within the shell. Altenbach et al. [21] developed a linear shell theory that accounts for transverse shear and surface stresses. Weicker et al. ([22] and [23]) developed a closed form solution for the static analysis of thin-walled pipes [22], and a finite element formulation [23]. Salahifar and Mohareb [24] formulated a closed form solution for circular cylindrical shells under harmonic forces. Amabili and Reddy [25] derived a consistent higher-order shear deformable theory for doubly curved shells of general geometries based on non-linear strain-displacement expressions. Their solution accounts for geometric imperfections. Paimushin [26] developed a large displacement shell theory based on the classical Kirchhoff-Love assumption. The solution accounts for deformations in the transverse direction by introducing additional displacement fields. Salahifar and Mohareb [27] formulated a finite element for the analysis of circular cylindrical thin shells under harmonic forces based on shape functions that satisfy the governing field equations. Favata and Podio-Guidugli [28] developed a theory of linearly elastic orthotropic shells with potential application to the continuous modeling of carbon nanotubes. Sansour et al. [29] developed a computationally efficiently strain gradient formulation that captures the scale effects of shell-like structures when one of the dimensions is very small relative to the other two dimensions. Xuea et al. [30] extended the KarmanDonnell theory for shallow cylindrical shells to account for large deformations. Based on an expansion of the axisymmetric equations of elasticity, Zozulya [31] developed a high-order theory for functionally graded axisymmetric cylindrical shells using Legendre polynomial series. Carrera et al. [32] (and [33]) developed a unified hierarchical formulation for multilayered composite structures, which enables the implementation of multiple plate/shell theories and finite elements based on a few fundamental nuclei. Cattabiani et al. [34] developed a variational shell theory that approximates the solution for the vibration problem as a sum of shape functions that identically satisfy the equilibrium equations while satisfying the weak form of the boundary conditions. Chowdhurya et al. [35] developed a state-based peridynamic formulation for the linear elastic analysis of shells that captures discontinuities by expressing the equations of motion in integro-differential form as opposed to partial differential equations. Zveryayev [36] developed an itertative shell solution, in which the three-dimensional equations of the elasticity in curvilinear coordinates were reduced using the Saint-Venant semi-inverse method. Awrejcewicz et al. [37] presented a mathematical model for nonlinear analysis of micro-shells that accounts for temperature-deformation coupling. 
94 theory for the free and transient vibration analysis of composite laminated cylindrical shells

95 by partitioning the radial displacement field into bending and shear components. Okhovat and

96 Bostrom [39] derived a hierarchy of shell equations in the form of power series in terms of the

97 shell thickness for the dynamic analysis of orthotropic cylindrical shells. Qingshan et al. [40]

98 developed a first-order shear deformable shell theory for the free and transient vibration

99 analysis of laminated open cylindrical shells with general boundary conditions.

\section{$100 \quad 1.2$ Common Assumptions}

101 In general, the theory of thin shells involves two aspects: a) developing the governing field 102 equations and the boundary conditions and b) providing solutions to the field equations for 103 problems with specific geometries, boundary conditions, and loading. Most thin-shell 104 formulations are based on the following assumptions:

105 1. Conservation of normals: All points lying on a normal to the middle surface before 106 deformation remain on the normal to the deformed middle surface. This implies a linear 107 distribution of the in-plane displacements across the thickness of the shell and zero shear 108 strains in the planes normal to the mid-surface.

109 2. A surface at a distance $z$ from the middle surface before deformation will remain at the 110 same distance from the middle surface after deformation, and

1113. Displacements are small compared to the radii of curvature of the middle surface. This 112 signifies that the curvatures of the shell mid-surface after deformation are considered 113 nearly equal to the curvatures before deformation.

\section{$114 \quad 1.3$ Differences among Shell Theories}

115 A survey of the literature reveals the presence of multiple cylindrical thin-shell theories. The

116 differences between these shell theories are attributed to the following aspects:

117 1. The adoption of different strain-displacement relationships: In some theories (e.g., 118 Timoshenko[1] and Flugge [3]) the strain-displacement relationships are obtained based on 119 geometric inspection of an infinitesimal portion of the shell undergoing deformation. In 120 other theories (e.g., Novozhilov [2], Saada [6] and Niordson [7]), a vector or tensor analysis 121 approach is adopted. 
2. The use of different methodologies in formulating the governing equations: In most theories (e.g., Timoshenko [1], Flugge [4], Saada [6] and Niordson [7]), equilibrium equations are obtained based on the force equilibrium of an infinitesimal shell element, while others (e.g., Novozhilov [2]) adopt a variational approach.

3. The enforcement of additional simplifications: Most researchers have applied various simplifications at different stages of the formulation, which had implications on the final form of the governing field equations and the boundary conditions. For example, Novozhilov [2] has neglected some of the terms in the expression of total potential energy compared to the other terms.

4. Inconsistencies in enforcing simplifications: Most theories (Timoshenko [1], Saada [6], Niordson [7], etc.) involved slight inconsistencies in their simplifications. For example, the effect of the shell thickness in the strain expression is neglected in some terms, but kept in other terms within the same formulation. These types of inconsistencies, in most cases, arose from separating the membrane strains from the bending strains.

Given the above discrepancies, it is difficult to judge which theories provide superior results and which are most suited for a given engineering problem. While, in some cases, there could be some qualitative notion on which theory could be judged to provide reliable predictions of the response, a systematic quantitative comparative assessment is missing. For a given problem, it becomes difficult to judge how much error is attributed to treating a given problem as a thin shell and how much is attributed to the approximations and treatments specific to the adopted theory. In order to elucidate the problem, the present treatment focuses on developing a generalized thin shell theory in which the strain-displacement relationships are thoroughly formulated based on tensor calculus in conjunction with assumptions that are well accepted within the framework of all thin-shell theories, while avoiding inconsistencies.

\section{Generalized Thin Shell Theory Formulation}

\subsection{Geometric preliminaries}

In general, a thin shell can be considered as a group of parallel curved surfaces in the immediate vicinity of the middle surface. Point $P_{0}$ on a surface can be defined by two curvilinear coordinates of a two dimensional subspace $\left(\mathbf{R}^{\alpha}\right)$ of the general three-dimensional 
space $\left(\mathbf{R}^{i}\right)$. As a notation convention, all Latin indices take the values 1, 2, 3, while Greek

152 indices take the values 1 and 2 . This implies that, in a curvilinear coordinate system $x^{i}$, the

153 geometry of any of those surfaces can be defined by two curvilinear coordinates $x^{\alpha}$ within

154 the middle surface and an additional coordinate $x^{3}$ normal to the middle surface (Figure 1).

155 In a Cartesian coordinate system $\chi^{i}$ with $\mathbf{I}_{i}$ as a covariant unit vector, position vector

$156 \mathbf{R}\left(\chi_{P_{1}}^{i}(t)\right)=\chi_{P_{1}}^{i}(t) \mathbf{I}_{i}$ at time $t$ for a point $P_{1}$ within the shell (Figure 1), can be defined as

157 the vector sum of position vector $\mathbf{r}\left(\chi_{P_{0}}^{i}(t)\right)=\chi_{P_{0}}^{i}(t) \mathbf{I}_{i}$ of its projection $P_{0}$ on the middle

158 surface and vector $\overrightarrow{P_{0} P_{1}}$, i.e.,

$$
\mathbf{R}\left(\chi_{P_{1}}^{i}(t)\right)=\mathbf{r}\left(\chi_{P_{0}}^{i}(t)\right)+\overrightarrow{P_{0} P_{1}}\left(\chi_{P_{0}}^{i}(t), \chi_{P_{1}}^{i}(t)\right)
$$

160 In Eq. 1, expressing the Cartesian coordinates $\chi^{i}$ for points $P_{0}$ and $P_{1}$ (i. e. $\chi_{P_{0}}^{i}(t)$ and $\chi_{P_{1}}^{i}(t)$

161 ) in terms of curvilinear coordinates $x^{i}$ enables expressing the position vectors for points $P_{0}$ 162 and $P_{1}$ as $\mathbf{r}\left(\chi_{P_{0}}^{i}(t)\right)=\chi^{i}\left(x_{P_{0}}^{j}, t\right) \mathbf{I}_{i}$ and $\mathbf{R}\left(\chi_{P_{1}}^{i}(t)\right)=\chi^{i}\left(x_{\AA}^{j}, t\right) \mathbf{I}_{i}$, respectively. Thus,

163 Cartesian coordinates $\chi^{i}$ appearing in Eq. 1 can be expressed in terms of curvilinear 164 coordinates $x^{i}$ yielding

$165 \quad \mathbf{R}\left(\chi^{i}\left(x_{\uparrow}^{j}, t\right)\right)=\mathbf{r}\left(\chi^{i}\left(x_{\curvearrowleft}^{j}, t\right)\right)+\overrightarrow{P_{0} P_{1}}\left(\chi^{i}\left(x_{\wp^{j}}^{j}, t\right), \chi^{i}\left(x_{\uparrow}^{j}, t\right)\right)$

166 or

$$
\mathbf{R}\left(\chi^{i}\left(x_{\uparrow_{1}^{j}}^{j}, t\right)\right)=\mathbf{r}\left(\chi^{i}\left(x_{r_{0}}^{\alpha}, t\right)\right)+x^{3} \mathbf{a}_{3}
$$

168 in which unit vector $\mathbf{a}^{3}=\left(\mathbf{a}_{1} \otimes \mathbf{a}_{2}\right) /\left|\mathbf{a}_{1} \otimes \mathbf{a}_{2}\right|$ is normal to the plane defined by base vectors $169 \mathbf{a}_{\alpha}=\partial \mathbf{r} / \partial x^{\alpha}$. Equation 3 provides the transformation of a shell surface from curvilinear 170 coordinate system $x^{i}$ into Cartesian coordinate system $\chi^{i}$. It can be re-written as

$$
\mathbf{R}\left(x^{1}, x^{2}, x^{3}, t\right)=\mathbf{r}\left(x^{1}, x^{2}, t\right)+x^{3} \mathbf{a}_{3}
$$

172 
173

174

175 In a similar manner, bases $\mathbf{g}_{i}=\partial \mathbf{R} / \partial x^{i}$ pertain to a general surface within the shell volume.

176 It can be shown that base vectors $\mathbf{g}_{\alpha}$ on a surface parallel to the mid-surface are related to the

177 base vectors of the middle surface $\mathbf{a}_{\gamma}$ through $\mathbf{g}_{\alpha}=\mu_{\alpha}^{\gamma} \mathbf{a}_{\gamma}$. Here, $\mu_{\alpha}^{\gamma}=\delta_{\alpha}^{\gamma}-x^{3} \bar{b}_{\alpha}^{\gamma}$ is the shifter

178 tensor, $\delta_{\alpha}^{\gamma}$ is the Kronecker delta tensor and $\bar{b}_{\alpha}^{\gamma}$ is the mixed curvature tensor for the middle

183 In general, the contravariant counterparts of the covariant base vectors are $\mathbf{g}^{\alpha}=\lambda_{\gamma}^{\alpha} \mathbf{a}^{\gamma}$ and

$184 \mathbf{g}^{3}=\mathbf{a}^{3}$, in which $\lambda_{\gamma}^{\alpha} \mu_{\beta}^{\gamma}=\delta_{\beta}^{\alpha}$ and the covariant and contravariant base vectors are related

185 through $\mathbf{g}_{j} \cdot \mathbf{g}^{i}=\delta_{j}^{i}$. The covariant and contravariant metric tensors for surfaces parallel to the

186 middle surface are respectively expressed as

187

$$
g_{\alpha \beta}=\mathbf{g}_{\alpha} \cdot \mathbf{g}_{\beta}=\mu_{\alpha}^{\gamma} \mu_{\beta}^{\delta} a_{\gamma \delta} \quad, \quad g^{\alpha \beta}=\mathbf{g}^{\alpha} \cdot \mathbf{g}^{\beta}=\lambda_{\gamma}^{\alpha} \lambda_{\delta}^{\beta} a^{\gamma \delta}
$$


188 where $a_{\gamma \delta}$ and $a^{\gamma \delta}$ are the covariant and contravariant metric tensors of the middle surface,

189 respectively, and defined as

190

$$
a_{\gamma \delta}=\mathbf{a}_{\gamma} \cdot \mathbf{a}_{\delta} \quad, \quad a^{\gamma \delta}=\mathbf{a}^{\gamma} \cdot \mathbf{a}^{\delta}
$$

191 The second fundamental tensor of the surface (curvature tensor) is defined as $\bar{b}_{\alpha \beta}=\mathbf{a}_{3, \alpha} \cdot \mathbf{a}_{\beta}$.

192 The Jacobian $g$ at a generic point within the shell, given by $g=\operatorname{det}\left(g_{i j}\right)$, represents a 193 differential element of volume.

$194 \quad 2.2$ Strains in Thin Shells

195 Using the Lagrangian approach, the strain tensor is defined in terms of the metric tensor

196 through $\eta_{i j}=\left(\hat{g}_{i j}-g_{i j}\right) / 2$. One can show [41] that the strain-displacement relationship is

197 defined by $\eta_{i j}=\frac{1}{2}\left(\left.U_{i}\right|_{j}+\left.U_{j}\right|_{i}+\left.\left.U^{k}\right|_{i} U_{k}\right|_{j}\right)$ in which, the notation $\left.\right|_{i}$ denotes the covariant

198 derivative of the argument vector with respect to $i=1,2,3$. Under the small deformation

199 assumption, the nonlinear terms $\left.\left.U^{k}\right|_{i} U_{k}\right|_{j}$ are negligible compared to the linear terms. Also,

200 for thin shells, the strains can be resolved into in-plane components $\eta_{\alpha \beta}$ and out-of-plane

201 components $\eta_{\alpha 3}=\eta_{3 \alpha}$ and $\eta_{33}$, and the displacement strain relations can be approximated by:

$202 \quad \eta_{\alpha \beta}=\frac{1}{2}\left(\left.U_{\alpha}\right|_{\beta}+\left.U_{\beta}\right|_{\alpha}\right), \eta_{\alpha 3}=\eta_{3 \alpha}=\frac{1}{2}\left(\left.U_{\alpha}\right|_{3}+\left.U_{3}\right|_{\alpha}\right), \quad \eta_{33}=\frac{1}{2}\left(\left.U_{3}\right|_{3}+\left.U_{3}\right|_{3}\right) \quad$ (7a-c $)$

203 According to Assumption 2 in Section 1.2 , the strain normal to the mid-surface vanishes, i.e.

$204 \eta_{33}=\frac{1}{2}\left(\left.U_{3}\right|_{3}+\left.U_{3}\right|_{3}\right) \simeq 0$. Also, according to Assumption 1 in Section 1.2 , the transverse 205 shear strains acting on the planes perpendicular to the mid-surface are negligible for thin shells 206 leading to the simplification $\eta_{\alpha 3}=\eta_{3 \alpha} \simeq 0$.

$207 \quad 2.3$ Displacements of a Point within the Shell

208 In general, displacement vector $\mathbf{U}$ of a point $A$ offset from the middle surface can be defined 209 as

$210 \quad \mathbf{U}=U_{i}\left(x^{1}, x^{2}, x^{3}, t\right) \mathbf{g}^{i}=U^{i}\left(x^{1}, x^{2}, x^{3}, t\right) \mathbf{g}_{i}$ 
211 where $U_{i}$ and $U^{i}$ are, respectively, the covariant and contravariant components of the 212 displacement vector in the $x^{i}$ coordinate system. Displacement vector $\mathbf{U}$ is related to position 213 vector $\mathbf{R}$ in the undeformed configuration (Figure 2) and position vector $\hat{\mathbf{R}}$ in the deformed 214 configuration through $\mathbf{U}=\hat{\mathbf{R}}-\mathbf{R}$. Vectors $\mathbf{R}$ and $\hat{\mathbf{R}}$ at point $A$ are related to vectors $\mathbf{r}$ and $215 \hat{\mathbf{r}}$ at the corresponding point $B$ on the mid-surface and unit vectors normal to the mid-surface $216 \mathbf{a}_{3}$ and $\hat{\mathbf{a}}_{3}$ as

$217 \quad \mathbf{U}=\hat{\mathbf{R}}-\mathbf{R}=\left(\hat{\mathbf{r}}+\hat{x}^{3} \hat{\mathbf{a}}_{3}\right)-\left(\mathbf{r}+x^{3} \mathbf{a}_{3}\right)=(\hat{\mathbf{r}}-\mathbf{r})+x^{3}\left(\hat{\mathbf{a}}_{3}-\mathbf{a}_{3}\right)$

218 Noting that the difference $\mathbf{u}=\hat{\mathbf{r}}-\mathbf{r}$ is the displacement vector at point $B$ and $\mathbf{a}_{3}, \hat{\mathbf{a}}_{3}$ are the 219 unit vectors normal to the mid-surface at point $B$ before and after deformation, the difference $220 \boldsymbol{\theta}=\hat{\mathbf{a}}_{3}-\mathbf{a}_{3}$ characterizes the angle of rotation of the middle surface and thus Eq. 9 can be re221 written as

$$
\mathbf{U}=\mathbf{u}\left(x^{1}, x^{2}, t\right)+x^{3} \boldsymbol{\theta}\left(x^{1}, x^{2}, t\right)
$$

223 Assumption 1 in Section 1.2, regarding the normality to the mid-surface, implies zero shear 224 strains on the planes perpendicular to the mid-surface, i.e. $\eta_{3 \alpha}=\eta_{\alpha 3}=\left(\left.U_{\alpha}\right|_{3}+\left.U_{3}\right|_{\alpha}\right) / 2=0$. 225 It can be used [41] to relate the displacement $\mathbf{U}$ of arbitrary point $A$, located at a distance $226 x^{3} \neq 0$ from the shell mid-surface within the shell, to those of its projection on the middle 227 surface, point $B\left(\mathbf{u}=u_{i} \mathbf{a}^{i}\right)$, through

$$
\mathbf{U}=U_{\alpha} \mathbf{g}^{\alpha}+U_{3} \mathbf{g}^{3} \quad ; \quad U_{\alpha}=\left(\mu_{\delta}^{\gamma} u_{\gamma}-x^{3} u_{3, \delta}\right) \mu_{\alpha}^{\delta} \quad, \quad U_{3}=u_{3}
$$

229 Figure 2 shows points $A$ and $B$, and their corresponding displacements $\mathbf{U}$ and $\mathbf{u}$, 230 respectively. The geometric interpretations for $U_{\alpha} \mathbf{g}^{\alpha}$ and $U_{3} \mathbf{g}^{3}$ are schematically shown in 231 Figure 3, in which point $A$ undergoes displacements $U_{\alpha}$ parallel to the shell mid-surface and 232 a displacement $U_{3}$ normal to the mid-surface. Similarly, the contravariant components of the 233 displacement are related to those of point $B$ on the middle surface through

$$
\mathbf{U}=U^{\alpha} \mathbf{g}_{\alpha}+U^{3} \mathbf{g}_{3} \quad, U^{\alpha}=U_{\beta} g^{\alpha \beta}=\left(\mu_{\delta}^{\gamma} u_{\gamma}-x^{3} u_{3, \delta}\right) \mu_{\beta}^{\delta} g^{\alpha \beta} \quad, U^{3}=u^{3}=u_{3}(12 \mathrm{a}-\mathrm{c})
$$




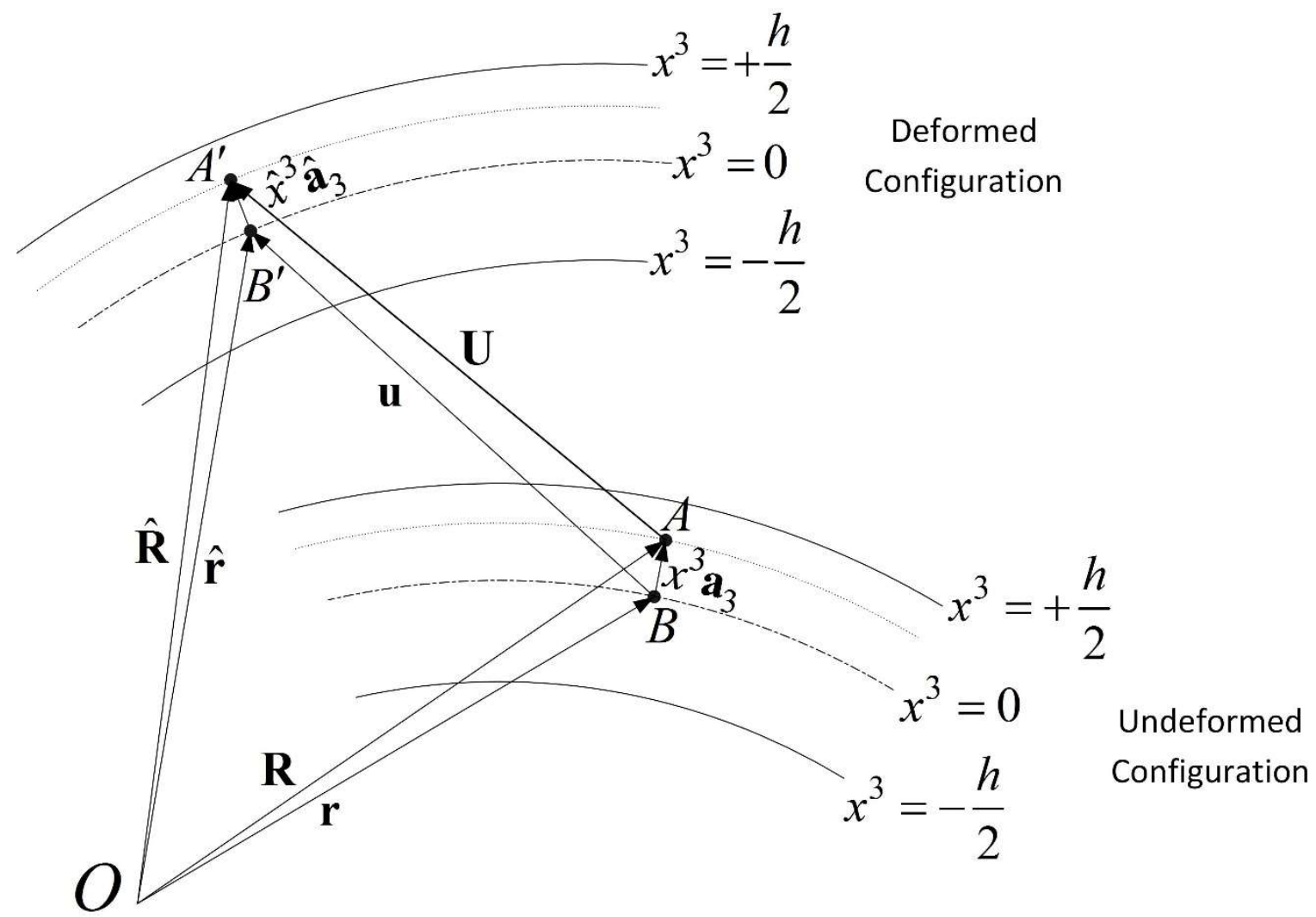

Figure 2- Deformed and Un-deformed Configurations of a Thin Shell

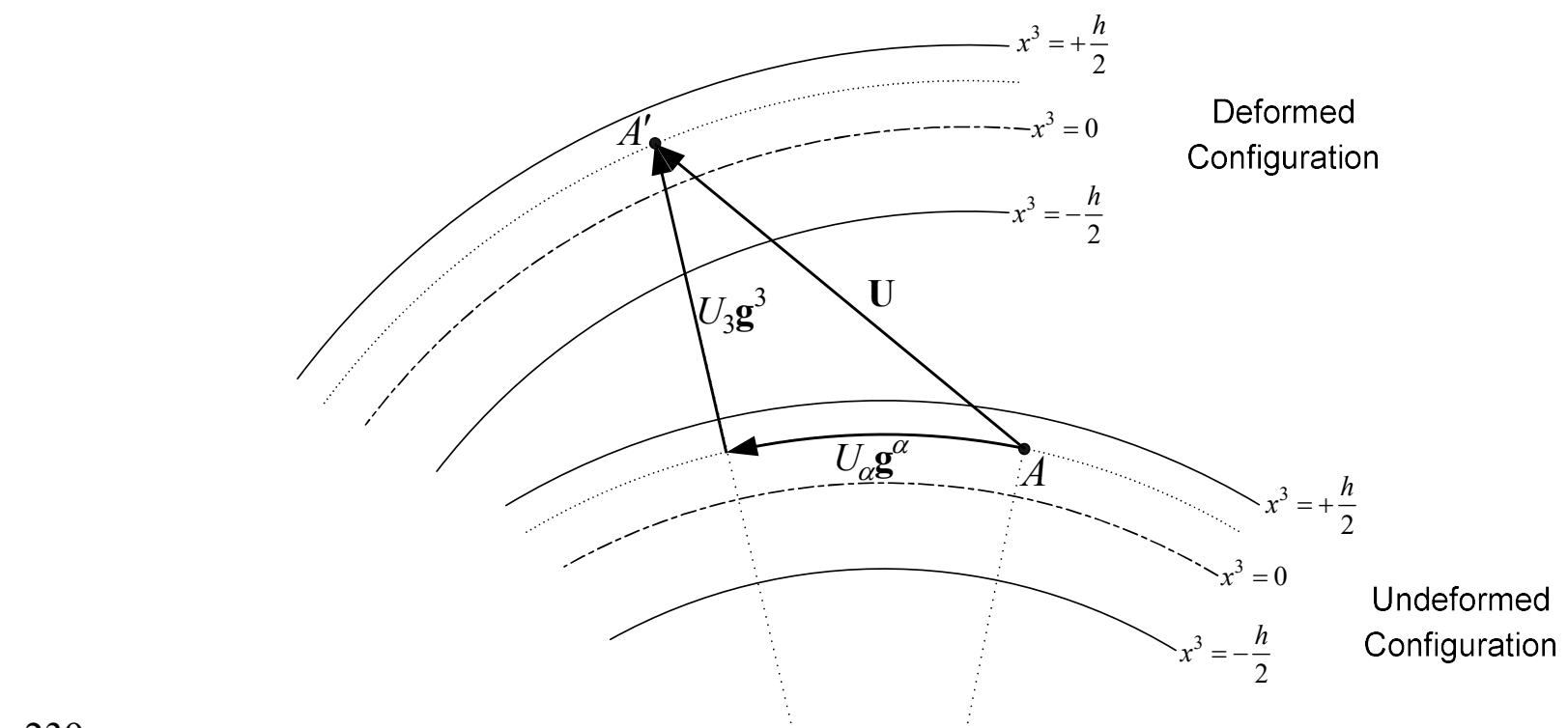




\subsection{Stress-Strain Relationship}

242 For elastic materials, stress tensor $\sigma^{i j}(i, j=1,2,3)$ for a point at a distance $x^{3}$ from the middle 243 surface $\left(-h / 2 \leq x^{3} \leq h / 2\right)$ is related to the corresponding strain tensor $\eta_{l m}(l, m=1,2,3)$

244 through $\sigma^{i j}=E^{i j l m} \eta_{l m}$ (e.g., Flugge [5]), in which $E^{i j l m}$ is the fourth order constitutive elastic 245 tensor. For thin shells, where the stresses perpendicular to the middle surface are negligible, 246 the number of non-zero stress components reduces from nine to four and the stress-strain 247 relationships reduce to

$$
\sigma^{\alpha \beta}=E^{\alpha \beta \lambda \delta} \eta_{\lambda \delta} \quad, \quad(\alpha, \beta, \lambda, \delta=1,2)
$$

249 Under linear elastic isotropy, the constitutive tensor can be shown [41] to take the form

$$
E^{\alpha \beta \lambda \delta}=\frac{E}{2(1+v)}\left[g^{\alpha \lambda} g^{\beta \delta}+g^{\alpha \delta} g^{\beta \lambda}+\frac{2 v}{1-v} g^{\alpha \beta} g^{\lambda \delta}\right]
$$

251 in which $E$ is the Modulus of elasticity and $v$ is Poisson's ratio.

\subsection{Hamilton Principle for Thin Shells of General Geometries}

253 According to Hamilton's principle, the variation $\delta$ П of the mechanical energy is given by

$254 \quad \int_{t_{1}}^{t_{2}} \delta \Pi d t=\int_{t_{1}}^{t_{2}} \delta\left(T^{*}-V^{*}\right) d t+\int_{t_{1}}^{t_{2}} \delta W^{*} d t=0$

255 in which all time integrations are performed over an arbitrary period starting from time $t_{1}$ to 256 time $t_{2}$ and $T^{*}, V^{*}$ and $W^{*}$ are the kinetic energy, internal strain energy and external work 257 in the system, respectively, and are given by

$258 \delta T^{*}=\int_{V} \rho \dot{U}^{i} \delta \dot{U}_{i} d V, \quad \delta V^{*}=\int_{V} E^{i j k l} \eta_{k l} \delta \eta_{i j} d V, \quad \delta W^{*}=\int_{V} F^{i} \delta U_{i} d V$ $(16 \mathrm{a}-\mathrm{c})$

259 in which integrations are over the volume $V$ and symbols $F^{i}$ denote the contravariant 260 components of the applied forces. From Eq. 16a-c, by substituting into Eq. 15, integrating by 261 parts the kinetic energy terms with respect to time, and using the relation $\left.262 U_{\alpha}\right|_{\beta}=U_{\alpha} \|_{\beta}-U_{3} b_{\alpha \beta}$ (in which, symbol $\|$ denotes the covariant derivative components of the 
263 preceding argument with respect to $x^{\alpha}$, i.e. $\left.U_{\alpha} \|_{\beta}=U_{\alpha, \beta}-U_{\gamma} \Gamma_{\alpha \beta}^{\gamma}\right)$ in the internal strain energy 264 integral term and integrating by parts with respect to $x^{\beta}$ yields [41]

$$
\begin{aligned}
\int_{t_{1}}^{t_{2}} \delta & \Pi d t=\left[\int_{V} \dot{U}^{i} \rho \delta U_{i} d V\right]_{t_{1}}^{t_{2}}+\int_{t_{1}}^{t_{2}}\left\{-\int_{V} \ddot{U}^{i} \rho \delta U_{i} d V d t\right. \\
& -\int_{A}\left[\frac{1}{2} E^{\alpha \beta \lambda \delta}\left(U_{\lambda}\left\|_{\delta}+U_{\delta}\right\|_{\lambda}-2 U_{3} b_{\lambda \delta}\right) \delta U_{\alpha}\right]_{0}^{x_{\beta}} d A \\
& +\int_{V}\left[\frac{1}{2} E^{\alpha \beta \lambda \delta}\left(U_{\lambda}\left\|_{\delta \beta}+U_{\delta}\right\|_{\lambda \beta}-2 U_{3, \beta} b_{\lambda \delta}-2 U_{3} b_{\lambda \delta} \|_{\beta}\right)\right. \\
& \left.+\frac{1}{2}\left(E^{\alpha \beta \lambda \delta} \|_{\beta}\right)\left(U_{\lambda}\left\|_{\delta}+U_{\delta}\right\|_{\lambda}-2 U_{3} b_{\lambda \delta}\right)\right] \delta U_{\alpha} d V \\
& +\int_{V} \frac{1}{2} E^{\alpha \beta \lambda \delta}\left(U_{\lambda}\left\|_{\delta}+U_{\delta}\right\|_{\lambda}-2 U_{3} b_{\lambda \delta}\right)\left(b_{\alpha \beta} \delta U_{3}\right) d V \\
& \left.+\int_{V} F^{i} \delta U_{i} d V\right\} d t=0
\end{aligned}
$$

266 Equation 17 is the variational form of the mechanical energy for a thin shell with general

267 curvature. The theory can be specialized to shells with specific geometries. In this context, the 268 present paper aims at specializing Eq. 17 to circular cylindrical shells. Readers interested in 269 the application of the theory to other shell geometries are referred to [41], where it has been 270 specialized to toroidal shells.

\section{$271 \quad 3 . \quad$ Special Case: Circular Cylindrical Thin Shells (CCTS)}

\subsection{Geometric properties}

273 Position vector $\mathbf{r}$ for a general point lying on the middle surface of a circular cylinder is 274 expressed in terms of unit vectors $\overrightarrow{\mathbf{I}}_{i}$ in the Cartesian coordinate system $\chi^{i}$ (Figure 4). In terms 275 of the curvilinear coordinates system $x^{i}$, it takes the form

$276 \quad \mathbf{r}\left(x^{1}, x^{2}, t\right)=x^{1} \overrightarrow{\mathbf{I}}_{1}+R \sin \left(x^{2} / R\right) \overrightarrow{\mathbf{I}}_{2}+R \cos \left(x^{2} / R\right) \overrightarrow{\mathbf{I}}_{3}$ 
279 From this point, symbols $(x, s, z)$ will be used instead of $\left(x^{1}, x^{2}, x^{3}\right)$ for clarity. The non-zero 280 281 282

Figure 4 - Cartesian and Curvilinear Coordinates components of the covariant and contravariant base vectors for a surface parallel to the midsurface lying within the shell can be shown [41] to be

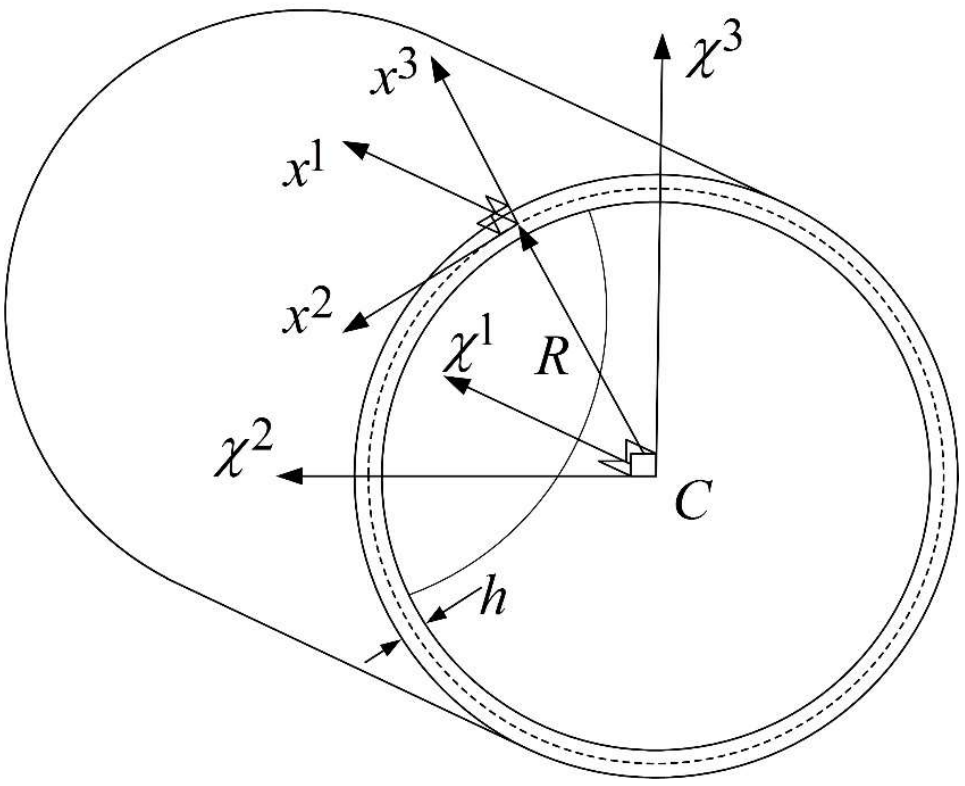

$$
\begin{array}{lll}
\mathbf{g}_{1}=\overrightarrow{\mathbf{j}}_{1}, & \mathbf{g}_{2}=(1+z / R) \overrightarrow{\mathbf{j}}_{2}, & \mathbf{g}_{3}=\overrightarrow{\mathbf{j}}_{3} \\
\mathbf{g}^{1}=\overrightarrow{\mathbf{j}}^{1}, & \mathbf{g}^{2}=(1+z / R)^{-1} \overrightarrow{\mathbf{j}}^{2}, & \mathbf{g}^{3}=\overrightarrow{\mathbf{j}}^{3}
\end{array}
$$

283 where $\overrightarrow{\mathbf{j}}_{i}$ and $\overrightarrow{\mathbf{j}}^{i}$ are the covariant and contravariant unit vectors associated with base vectors $\mathbf{g}_{i}$ and $\mathbf{g}^{i}$, respectively. The non-zero covariant and contravariant components of the metric 285 tensor are

$$
g_{11}=g_{33}=g^{11}=g^{33}=1, g_{22}=(1+z / R)^{2}, g^{22}=(1+z / R)^{-2}
$$

287 Also, the non-zero covariant and mixed components of the curvature tensor for a general 288 surface within the shell that is parallel to the middle surface are $b_{22}=-(1+z / R) / R$ and $289 b_{2}^{2}=-1 / R(1+z / R)$, respectively. Finally, the volume of an infinitesimal volumetric element 290 is $d V=(1+z / R) d z d s d x$. 


\subsection{Displacements in terms of mid-surface displacements}

292 Considering the geometric properties obtained in Section 3.1, the covariant and contravariant 293 displacement components are expressed as

$$
\begin{array}{lll}
U_{1}=u-z w_{, 1} & , U_{2}=(1+z / R)^{2} v-z(1+z / R) w_{, 2}, & U_{3}=w \\
U^{1}=u-z w_{, 1} & , U^{2}=v-z(1+z / R)^{-1} w_{, 2} & , U^{3}=w
\end{array}
$$

295 where symbols $(u, v, w)$ have been used instead of $\left(u_{1}, u_{2}, u_{3}\right)$ for clarity. It is noted that the 296 covariant and contravariant components of the displacements become identical at the middle 297 surface $z=0$, i.e., $U_{i}(z=0)=U^{i}(z=0)$.

\section{$298 \quad 3.3$ Strain-displacement relations}

299 By specializing the strain displacement relations in Eq. 7a, to cylindrical shells (e.g., [41]) one 300 obtains

$$
\eta_{\alpha \beta}=\frac{1}{2}\left(\mu_{\alpha}^{\delta} \mu_{\delta}^{\gamma} u_{\gamma, \beta}-z \mu_{\alpha}^{\delta} u_{3, \delta \beta}-\mu_{\alpha}^{\gamma} u_{3} \bar{b}_{\gamma \beta}+\mu_{\beta}^{\delta} \mu_{\delta}^{\gamma} u_{\gamma, \alpha}-z \mu_{\beta}^{\delta} u_{3, \delta \alpha}-\mu_{\beta}^{\gamma} u_{3} \bar{b}_{\gamma \alpha}\right)
$$

302 Prescribing the shifter tensor $\mu_{\alpha}^{\delta}$ and the curvature tensor of the middle surface $\bar{b}_{\gamma \beta}$, introduced 303 in Sec. 2.1, for CCTSs and substituting into Eq. 22 yields the corresponding covariant strain 304 tensor. The physical components of the strain tensor, denoted subsequently by a tilda, can be 305 expressed as

$$
\tilde{\eta}_{11}=u_{, 1}-z w_{, 11}
$$

$$
\begin{aligned}
& \tilde{\eta}_{22}=v_{, 2}+\frac{w}{R(1+z / R)}-\frac{z w_{, 22}}{(1+z / R)} \\
& \tilde{\eta}_{12}=\tilde{\eta}_{21}=\frac{1}{2}\left\{\frac{u_{, 2}}{(1+z / R)}+(1+z / R) v_{, 1}-z\left[1+\frac{1}{(1+z / R)}\right] w_{, 12}\right\}
\end{aligned}
$$

\subsection{Components of Constitutive Tensor}

308 Given the components of the metric tensors (Eqs.20), by substituting into the elastic 309 constitutive tensor in Eq.14, one recovers the following expressions for non-zero components 310 of the constitutive tensor 
311

$$
\begin{aligned}
& E^{1111}=\frac{E}{1-v^{2}}, \quad E^{2222}=\frac{E}{\left(1-v^{2}\right)(1+z / R)^{4}}, \quad E^{1122}=E^{2211}=\frac{E v}{\left(1-v^{2}\right)(1+z / R)^{2}}, \\
& E^{1212}=E^{1221}=E^{2112}=E^{2121}=\frac{E(1-v)}{2\left(1-v^{2}\right)(1+z / R)^{2}}
\end{aligned}
$$

\section{$313 \quad 3.5$ Hamilton Principle for CCTS}

314 From Eqs. 21 and 24, by substituting into the variation of the Hamilton's principle in Eq. 17, 315 integrating with respect to $z$, grouping like-terms and integrating by parts with respect to $x$ 316 and $s$ yields [41]

$$
\begin{aligned}
& \int_{t_{1}}^{t_{2}} \delta \Pi d t=\int_{t_{1}}^{t_{2}}\left\langle\int _ { s } \frac { E h } { 1 - v ^ { 2 } } \left[\left({ }_{1}^{1} \bar{H} u_{, 1}+{ }_{2}^{1} \bar{H} v_{, 2}+{ }_{3}^{1} \bar{H} w+{ }_{4}^{1} \bar{H} w_{, 11}\right) \delta u\right.\right. \\
&+\left({ }_{1}^{2} \bar{H} u_{, 2}+{ }_{2}^{2} \bar{H} v_{, 1}+{ }_{3}^{2} \bar{H} w_{, 12}\right) \delta v \\
&+\left({ }_{1}^{3} \bar{H} u_{, 11}+{ }_{2}^{3} \bar{H} u_{, 22}+{ }_{3}^{3} \bar{H} v_{, 12}+{ }_{4}^{3} \bar{H} w_{, 111}+{ }_{5}^{3} \bar{H} w_{, 122}+{ }_{6}^{3} \bar{H} \ddot{u}+{ }_{7}^{3} \bar{H} \ddot{w}_{, 1}+{ }_{8}^{3} \bar{H} f^{x w}\right) \delta w \\
&\left.+\left({ }_{1}^{4} \bar{H} u_{, 1}+{ }_{2}^{4} \bar{H} v_{, 2}+{ }_{3}^{4} \bar{H} w_{, 11}+{ }_{4}^{4} \bar{H} w_{, 22}\right) \delta w_{, 1}\right]_{0}^{L} d s \\
&+\int_{A} \frac{E h}{1-} v^{2}\left[\left({ }_{1}^{1} \bar{G} u_{, 11}+{ }_{2}^{1} \bar{G} u_{, 22}+{ }_{3}^{1} \bar{G} v_{, 12}+{ }_{4}^{1} \bar{G} w_{, 1}+{ }_{5}^{1} \bar{G} w_{, 111}+{ }_{6}^{1} \bar{G} w_{, 122}\right.\right. \\
&+\left({ }_{1}^{2} \bar{G} u_{, 12}+{ }_{2}^{2} \bar{G} v_{, 11}+{ }_{3}^{2} \bar{G} v_{, 22}+{ }_{4}^{2} \bar{G} w_{, 2}+{ }_{5}^{2} \bar{G} w_{, 112}+{ }_{a}^{2} \bar{G} w_{, 222}+{ }_{6}^{2} \bar{G} \ddot{v}+{ }_{7}^{2} \bar{G} \ddot{w}_{, 2}+{ }_{8}^{2} \bar{G} f^{s v}\right) \delta v \\
&+\left({ }_{1}^{3} \bar{G} u_{, 1}+{ }_{2}^{3} \bar{G} v_{, 2}+{ }_{3}^{3} \bar{G} w+{ }_{a}^{3} \bar{G} w_{, 11}+{ }_{4}^{3} \bar{G} w_{, 22}+{ }_{5}^{3} \bar{G} u_{, 111}+{ }_{6}^{3} \bar{G} u_{, 122}+{ }_{7}^{3} \bar{G} v_{, 112}+{ }_{b}^{3} \bar{G} w_{, 222}\right. \\
&+{ }_{8}^{3} \bar{G} w_{, 1111}+{ }_{9}^{3} \bar{G} w_{, 1122}+{ }_{10}^{3} \bar{G} w_{, 2222}+{ }_{11}^{3} \bar{G} \ddot{w}+{ }_{12}^{3} \bar{G} \ddot{w}_{, 11}+{ }_{13}^{3} \bar{G} \ddot{w}_{, 22}+{ }_{14}^{3} \bar{G} \ddot{u}_{, 1}+{ }_{15}^{3} \bar{G} \ddot{v}_{, 2} \\
&\left.\left.\left.\quad+{ }_{16}^{3} \bar{G} f_{, 1}^{x w}+{ }_{17}^{3} \bar{G} f_{, 2}^{s w}+{ }_{18}^{3} \bar{G} f z w\right) \delta w\right] d s d x\right\rangle d t=0
\end{aligned}
$$

318 where coefficients ${ }_{1}^{1} \bar{G}-{ }_{18}^{3} \bar{G}$ and ${ }_{1}^{1} \bar{H}-{ }_{4}^{4} \bar{H}$, depend on the parameter

$$
\Phi=\Phi(h / R)=(R / h) \ln [(2+h / R) /(2-h / R)]
$$

and are defined in Appendix A. The terms $f^{x u}, f^{x w}, f^{s v}, f^{s w}, f^{z w}$ arising in Eq. 25 are related to the physical force components per unit volume $p^{x}, p^{s}, p^{z}$ and are provided in Appendix B. 
324 The field equations and corresponding boundary conditions for a CCTS can be obtained from 325 Eq. 25 through integration by parts. The details and relevant equations are provided in 326 Appendix B, where the boundary conditions are given by Eq. B.2 and the field equations are 327 given by Eq. B.3. These are entirely consistent with the assumptions postulated in Section 1.2 328 . In order to recover simpler approximate forms of the field equations and cast the present 329 formulation in a format comparable to other CCTS theories, the logarithmic function (Eq. 26

330 ) appearing in Eq. 25 is replaced by a truncated Taylor series expansion $\Phi(h / R) \approx 1+(h / R)^{2} / 12+(h / R)^{4} / 80+\cdots$

332 By omitting all time dependent terms, retaining the traction terms $f^{x u}, f^{s v}, f^{z w}$, omitting 333 the terms $f^{x w}, f^{s w}$ and using the series approximation in Eq. 27 up to the second term, one 334 recovers the field equations in Flugge's theory [4] as a special case of the present formulation. 335 A simpler version of the present theory can also be obtained by adopting the approximation $3361+z / R \approx 1$, in the strain displacement expressions (Eqs. 23a-c), the base vector expressions, 337 and the Jacobian. The resulting field equations are found to coincide with those of the DMV 338 theory [7]. Finally, as $(1+z / R) \rightarrow 1$, the present theory, the Flugge theory and the DMV are 339 found to converge to the same field equations.

340 In contrast to other thin-shell theories known to the authors, the present formulation treats the 341 applied loads as body forces, and thus, is able to capture the spatial variations of the loads 342 along all three dimensions. Mid-surface tractions, line loads, and point loads can all be treated 343 as body forces by using the Dirac delta and Heaviside functions.

\subsection{Comparison}

345 Table 1 compares the strain expressions adopted in the past theories to those of the present 346 study. All theories are in agreement regarding the expression $\tilde{\eta}_{11}=u_{, 1}-z w_{, 11}$ for the 347 longitudinal strain. The Jacobian determinant is taken as unity in all theories except the present 348 and Flugge's theories, in which the Jacobian is $(1+z / R)^{2}$. Table 1 provides a comparison of 349 the circumferencial and shear strains $\tilde{\eta}_{22}$ and $\tilde{\eta}_{12}$ as given in various theories. Strain 
expressions based on Flugge's theory are observed to become in exact agreement with those

351 of the present theory when the approximation $\Phi(h / R) \approx 1+(h / R)^{2} / 12$ is adopted. The

352 Novozhilov strain expressions can be obtained from the strains of the present theory by 353 applying the approximations $\Phi(h / R) \approx 1+(h / R)^{2} / 12$ and $1+z / R \approx(1-z / R)^{-1}$, while the

354 DMV strain expressions can be recovered from the strains of the present theory by applying 355 the approximations $\Phi(h / R) \approx 1+(h / R)^{2} / 12$ and $1+z / R \approx 1$. In contrast, strain expressions 356 in the theories of Timoshenko [1], Saada [6], Morley-Koiter [7] and Niordson [7] include 357 additional terms that do not arise in the present tensor-based approach. Such terms stem from additional approximations that have been introduced in these theories, but avoided in the present tensorial treatment.

Table 1-Comparing strain-displacement expressions in various thin shell theories

\begin{tabular}{|c|c|c|}
\hline \multirow[t]{2}{*}{ Theory } & \multicolumn{2}{|c|}{ Strain Expressions } \\
\hline & $\tilde{\eta}_{22}$ & $\tilde{\eta}_{12}$ \\
\hline $\begin{array}{l}\text { Flugge's theory }[4] \& \\
\text { Present Theory } \\
\text { with approximation } \\
\Phi(h / R) \approx 1+(h / R)^{2} / 12\end{array}$ & $v_{, 2}+\frac{1}{R+z} w-z \frac{R}{R+z} w_{, 22}$ & $\frac{1}{2}\left(\frac{R}{R+z} u_{, 2}+\frac{R+z}{R} v_{, 1}-z \frac{2 R+z}{R+z} w_{, 12}\right.$ \\
\hline $\begin{array}{l}\text { Novozhilov [2] \& } \\
\text { Present Theory with } \\
\text { approximations } \\
\Phi(h / R) \approx 1+(h / R)^{2} / 12 \\
\text { and } 1+z / R \approx(1-z / R)^{-1}\end{array}$ & $v_{, 2}+\frac{1}{R} \frac{R-z}{R} w-z w_{, 22}$ & $\frac{1}{2}\left(\frac{R-z}{R} u_{, 2}+\frac{R+z}{R} v_{, 1}-2 z w_{, 12}\right)$ \\
\hline Saada [6] & $\frac{R+z}{R} v_{, 2}+\frac{1}{R} w-z w_{, 22}$ & $\frac{1}{2}\left(u_{, 2}+\frac{R+z}{R} v_{, 1}-2 z w_{, 12}\right)$ \\
\hline Timoshenko [1] & $\frac{R+z}{R} v_{, 2}+\frac{1}{R} w-z w_{, 22}$ & $\frac{1}{2}\left(u_{, 2}+\frac{R+2 z}{R} v_{, 1}-2 z w_{, 12}\right)$ \\
\hline $\begin{array}{l}\text { Morley-Koiter [7] \& } \\
\text { Niordson [7] }\end{array}$ & $\frac{R+2 z}{R} v_{, 2}+\frac{1}{R} \frac{R+z}{R} w-z w_{, 22}$ & $\frac{1}{2}\left(u_{, 2}+\frac{R+2 z}{R} v_{, 1}-2 z w_{, 12}\right)$ \\
\hline $\begin{array}{l}\text { DMV [7] \& } \\
\text { Present Theory } \\
\text { with approximations } \\
\Phi(h / R) \approx 1 \text { and } \\
1+z / R \approx 1\end{array}$ & $v_{, 2}+\frac{1}{R} w-z w_{, 22}$ & $\frac{1}{2}\left(u_{, 2}+v_{, 1}-2 z w_{, 12}\right)$ \\
\hline
\end{tabular}

361 The presence of such differences at the strain expression stage, combined with other 362 simplifications and approximations, leads to differences in their corresponding field equations 
and boundary conditions. In order to conduct a comparative study, irrespective of the CCTS

364 theory adopted, the equilibrium conditions can be cast into the following generic form

$$
\begin{aligned}
u_{, 11}+\alpha_{1.1} \frac{1-v}{2} u_{, 22}+\frac{1+v}{2} v_{, 12}+\frac{v}{R} w_{, 1}-\alpha_{1.2} \frac{h^{2}}{12 R} w_{, 111}+\alpha_{1.3} \frac{1-v}{2} w_{, 122} \\
-\rho \frac{1-v^{2}}{E}\left(\ddot{u}-\alpha_{1.4} \frac{h^{2}}{12 R} \ddot{w}_{, 1}\right)+f^{x u}=0
\end{aligned}
$$

$$
\frac{1+v}{2} u_{, 12}+\alpha_{2.1} \frac{1-v}{2} v_{, 11}+\alpha_{2.2} v_{, 22}+\frac{1}{R} w_{, 2}-\alpha_{2.3} \frac{h^{2}}{12 R} w_{, 112}-\alpha_{2.4} \frac{h^{2}}{12 R} w_{, 222}
$$

$$
-\rho \frac{1-v^{2}}{E}\left(\alpha_{2.5} \ddot{v}-\alpha_{2.6} \frac{h^{2}}{6 R} \ddot{w}_{, 2}\right)+f^{s v}=0
$$

$$
\frac{v}{R} u_{, 1}+\frac{1}{R} v_{, 2}+\alpha_{3.1} \frac{1}{R^{2}} w+\alpha_{3.2} w_{, 11}+\alpha_{3.3} w_{, 22}-\alpha_{3.4} \frac{h^{2}}{12 R} u_{, 111}+\alpha_{3.5} \frac{1-v}{2} u_{, 122}
$$

$$
-\alpha_{3.6} \frac{h^{2}}{12 R} v_{, 112}+\alpha_{3.7} w_{, 222}+\frac{h^{2}}{12} w_{, 1111}+\alpha_{3.8} w_{, 1122}+\alpha_{3.9} w_{, 2222}
$$

$$
\begin{array}{r}
-\rho\left(1-v^{2}\right) / E\left[\ddot{w}-\alpha_{3.10} \frac{h^{2}}{12}\left(\ddot{w}_{, 11}+\ddot{w}_{, 22}\right)+\alpha_{3.11} \frac{h^{2}}{12 R}\left(\ddot{u}_{, 1}+2 \ddot{v}_{, 2}\right)\right] \\
+\left(1-v^{2}\right) / E h\left(-f_{, 1}^{x w}-f_{, 2}^{s w}+f^{z w}\right)=0
\end{array}
$$

368 where coefficients $\alpha_{i . j}$ are theory-dependent and are defined in Table 2. As observed, the adoption of various assumptions/approximations in various theories, has led to the elimination of terms in some cases (e.g., $-\alpha_{1.2}\left(h^{2} / 12 R\right) w_{, 111}, \quad-\alpha_{3.4}\left(h^{2} / 12 R\right) u_{, 111} \quad$ and $\alpha_{3.5}[(1-v) / 2] u_{, 122}$ for Timoshenko, Novozhilov and Saada), and to the emergence of 372 additional terms in other cases (e.g., $\alpha_{2.4} w_{, 222}, \alpha_{3.2} w_{, 11}$ and $\alpha_{3.7} w_{, 222}$ for Timoshenko, 373 Novozhilov and Saada), when compared to the present treatment. Such approximations/simplifications have only affected the magnitude of coefficients of other terms in some cases.

Table 2 - Comparison of field equation coefficients $\alpha_{i . j}$ for various CCTS theories

\begin{tabular}{|c|c|c|c|c|}
\hline F.E.s & Present Theory & Flugge [4] & $\begin{array}{c}\text { Timoshenko [1], Novozhilov [2], } \\
\text { Saada [6], }\end{array}$ & $\begin{array}{c}\text { Morley-Koiter [7], } \\
\text { Niordson [7], DMV } \\
\text { [7] }\end{array}$ \\
\hline$\alpha_{1.1}$ & $\Phi$ & $\left(1+h^{2} / 12 R^{2}\right)$ & 1 & 1 \\
\hline$\alpha_{1.2}$ & 1 & 1 & 0 & 0 \\
\hline
\end{tabular}




\begin{tabular}{|c|c|c|c|c|}
\hline$\alpha_{1.3}$ & $R(\Phi-1)$ & $h^{2} / 12 R$ & 0 & 0 \\
\hline$\alpha_{1.4}$ & 1 & - & 0 & 0 \\
\hline$\alpha_{2.1}$ & $1+h^{2} / 4 R^{2}$ & $1+h^{2} / 4 R^{2}$ & $\begin{array}{c}1+h^{2} / 3 R^{2} \\
{\left[\text { Take }\left(1+h^{2} / 12 R^{2}\right) \text { for Timoshenko] }\right.}\end{array}$ & 1 \\
\hline$\alpha_{2.2}$ & 1 & 1 & $1+h^{2} / 12 R^{2}$ & 1 \\
\hline$\alpha_{2.3}$ & $(3-v) / 2$ & $(3-v) / 2$ & $\begin{array}{c}1 \\
{[\text { Take }(2-v) \text { for Novozhilov] }}\end{array}$ & 0 \\
\hline$\alpha_{2.4}$ & 0 & 0 & 1 & 0 \\
\hline$\alpha_{2.5}$ & $1+h^{2} / 12 R^{2}$ & $1+h^{2} / 12 R^{2}$ & - & 1 \\
\hline$\alpha_{2.6}$ & 1 & 1 & - & 0 \\
\hline$\alpha_{3.1}$ & $\Phi$ & $1+h^{2} / 12 R^{2}$ & 1 & $\begin{array}{c}1+h^{2} / 12 R^{2} \\
{[\text { Take unity for DMV] }}\end{array}$ \\
\hline$\alpha_{3.2}$ & 0 & 0 & 0 & $\begin{array}{c}1+h^{2} / 6 R^{2} \\
\text { [Vanishes for DMV] }\end{array}$ \\
\hline$\alpha_{3.3}$ & $2(\Phi-1)$ & $h^{2} / 6 R^{2}$ & 0 & $\begin{array}{c}h^{2} / 6 R^{2} \\
\text { [Vanishes for DMV] }\end{array}$ \\
\hline$\alpha_{3.4}$ & 1 & 1 & 0 & 0 \\
\hline$\alpha_{3.5}$ & $R(\Phi-1)$ & $h^{2} / 12 R$ & 0 & 0 \\
\hline$\alpha_{3.6}$ & $(3-v) / 2$ & $(3-v) / 2$ & $\begin{array}{c}(2-v) \\
\text { [Take unity for Saada] }\end{array}$ & 0 \\
\hline$\alpha_{3.7}$ & 0 & 0 & $h^{2} / 12 R$ & 0 \\
\hline$\alpha_{3.8}$ & $\begin{array}{c}(3+v) h^{2} / 24+ \\
R^{2}(\Phi-1)(1-v) / 2 \\
\end{array}$ & $h^{2} / 6$ & $h^{2} / 6$ & $h^{2} / 6$ \\
\hline$\alpha_{3.9}$ & $R^{2}(\Phi-1)$ & $h^{2} / 12$ & $h^{2} / 12$ & $h^{2} / 12$ \\
\hline$\alpha_{3.10}$ & 1 & - & - & 1 \\
\hline$\alpha_{3.11}$ & 1 & - & - & 0 \\
\hline$\alpha_{3.12}$ & 1 & - & - & 1 \\
\hline
\end{tabular}

378 Similarly, the boundary terms have been cast into the following generic form.

379

$$
\left(u_{, 1}+v v_{, 2}+\frac{v}{R} w-\beta_{1.1} \frac{h^{2}}{12 R} w_{, 11}\right) \delta u
$$

$$
\left(u_{, 2}+\beta_{2.1} v_{, 1}-\frac{h^{2}}{4 R} w_{, 12}\right) \delta v
$$


$\left(\beta_{3.1} \frac{h^{2}}{12 R} u_{, 11}-\beta_{3.2} \frac{1-v}{2} u_{, 22}+\beta_{3.3} \frac{h^{2}}{12 R} v_{, 12}-\frac{h^{2}}{12} w_{, 111}-\beta_{3.4} \frac{h^{2}}{12} w_{, 122}\right) \delta w$

$\left(\beta_{4.1} \frac{h^{2}}{12 R} u_{, 1}+\beta_{4.2} \frac{h^{2}}{12 R} v v_{, 2}-\frac{h^{2}}{12} w_{, 11}-\frac{h^{2}}{12} v w_{, 22}\right) \delta w_{, 1}$

383 Table 3 provides coefficients $\beta_{i . j}$ for the present theory and those of Flugge, Timoshenko 384 and DMV.

385

386

Table 3 - Comparison of field equation coefficients $\beta_{i . j}$ for various CCTS theories

\begin{tabular}{|c|c|c|c|c|}
\hline B.C.s & Present Theory & Flugge & Timoshenko & DMV \\
\hline$\beta_{1.1}$ & 1 & 1 & 0 & 0 \\
\hline$\beta_{2.1}$ & $\left(1+h^{2} / 4 R^{2}\right)$ & $\left(1+h^{2} / 4 R^{2}\right)$ & $2 / 3$ & 0 \\
\hline$\beta_{3.1}$ & 1 & 1 & 0 & 0 \\
\hline$\beta_{3.2}$ & $R(\Phi-1)$ & $h^{2} / 12 R$ & 0 & 0 \\
\hline$\beta_{3.3}$ & $(3-v) / 2$ & 1 & 1 & 0 \\
\hline$\beta_{3.4}$ & {$\left[(3-v) / 2+(\Phi-1)(1-v) 6 R^{2} / h^{2}\right]$} & $(2-v)$ & 1 & $(2-v)$ \\
\hline$\beta_{4.1}$ & 1 & 1 & 1 & 0 \\
\hline$\beta_{4.2}$ & 1 & & & 0 \\
\hline
\end{tabular}

\section{$388 \quad 3.8$ Steady State Analysis under Harmonic Forces}

389 Consider a CCTS under general harmonic force per unit volume $\left[p^{x}(x, s, z, t), p^{s}(x, s, z, t), p^{z}(x, s, z, t)\right]=\left[\bar{p}^{x}(x, s, z), \bar{p}^{s}(x, s, z), \bar{p}^{z}(x, s, z)\right] \operatorname{Re}\left(e^{i \bar{\omega} t}\right)$,

391 with an exciting frequency $\bar{\omega}$. It is possible to express the force functions using double Fourier 392 series with $N$ circumferential modes and $K$ longitudinal modes yielding: 


$$
\left\{\begin{array}{l}
p^{x} \\
p^{s} \\
p^{z}
\end{array}\right\}=\sum_{n=-N}^{N} \sum_{k=-K}^{K}\left[\left\{\begin{array}{c}
p_{n k}^{x}(z) \\
p_{n k}^{s}(z) \\
p_{n k}^{z}(z)
\end{array}\right\} e^{2 \pi i k x / L} e^{i n s / R}\right] \operatorname{Re}\left(e^{i \bar{\omega} t}\right)
$$

393

$$
\left\{\begin{array}{c}
p_{n k}^{x}(z) \\
p_{n k}^{s}(z) \\
p_{n k}^{z}(z)
\end{array}\right\}=\int_{s=0}^{s=2 \pi R} \int_{x=0}^{x=L}\left\{\begin{array}{l}
\bar{p}^{x}(x, s, z) \\
\bar{p}^{s}(x, s, z) \\
\bar{p}^{z}(x, s, z)
\end{array}\right\} e^{-2 \pi i k x / L} e^{-i n s / R} d x d s
$$

394 in which, each applied force component is expressed in terms of its Fourier series expansion 395 in $x$ and $s$. From Eq. 28, by substituting into Equations B.1a-e and integrating over the 396 thickness, one obtains

$$
\left\{\begin{array}{l}
f^{x u} \\
f^{s v} \\
f^{z w} \\
f^{x w} \\
f^{s w}
\end{array}\right\}=\left[\sum_{n=-N}^{N} \sum_{k=-K}^{K}\left\{\begin{array}{l}
f_{n k}^{x u} \\
f_{n k}^{s v} \\
f_{n k}^{z w} \\
f_{n k}^{x w} \\
f_{n k}^{s w}
\end{array}\right\} e^{2 \pi i k x / L} e^{i n s / R}\right] \operatorname{Re}\left(e^{i \bar{\omega} t}\right)
$$

397

$$
\left\{\begin{array}{l}
f_{n k}^{x u} \\
f_{n k}^{s v} \\
f_{n k}^{z w} \\
f_{n k}^{x w} \\
f_{n k}^{s w}
\end{array}\right\}=\int_{z=-\frac{h}{2}}^{z=+\frac{h}{2}}\left\{\begin{array}{c}
p_{n k}^{x}(z)(1+z / R) \\
p_{n k}^{s}(z)(1+z / R)^{2} \\
p_{n k}^{z}(z)(1+z / R) \\
p_{n k}^{x}(z) z(1+z / R) \\
p_{n k}^{z}(z) z(1+z / R)
\end{array}\right\} d z
$$

398 For a given load, constants $f^{x u}, f^{s v}, f^{z w}, f^{x w}$ and $f^{s w}$ are determined and a procedure 399 similar to that reported in [24] is then used to provide a closed form solution for each of the 400 theories developed. The corresponding steady state displacements are expressed in the 401 following form. 


$$
\begin{aligned}
& u(x, s, t)=\bar{u}(x, s) e^{i \bar{\omega} t}=\sum_{n=-N}^{N}\left(\sum_{j=1}^{8} A_{n j} \bar{A}_{n j} e^{m_{n j} x}+\sum_{k=-K}^{K} R_{n k} e^{2 \pi i k x / L}\right) e^{i n s / R} e^{i \bar{\omega} t} \\
& v(x, s, t)=\bar{v}(x, s) e^{i \bar{\omega} t}=\sum_{n=-N}^{N}\left(\sum_{j=1}^{8} A_{n j} \bar{B}_{n j} e^{m_{n j} x}+\sum_{k=-K}^{K} S_{n k} e^{2 \pi i k x / L}\right) e^{i n s / R} e^{i \bar{\omega} t} \\
& w(x, s, t)=\bar{w}(x, s) e^{i \bar{\omega} t}=\sum_{n=-N}^{N}\left(\sum_{j=1}^{8} A_{n j} \bar{C}_{n j} e^{m_{n j} x}+\sum_{k=-K}^{K} T_{n k} e^{2 \pi i k x / L}\right) e^{i n s / R} e^{i \bar{\omega} t}
\end{aligned}
$$

403 Symbols $m_{n j}$ are the eigenvalues and each set of symbols $\bar{A}_{n j}, \bar{B}_{n j}, \bar{C}_{n j}$ form the corresponding eigenvectors of the characteristic equation of the system related to mode $n$.

405 Symbols $R_{n k}, S_{n k}, T_{n k}$ and $A_{n j}$ are unknown integration constants to be determined from 406 boundary conditions (e.g., [24]).

\subsection{Examples}

408 In the following examples, the above solution technique is adopted to express the applied 409 forces and displacement fields in the circumferential direction $s$ and lomgitudinal direction $x$ 410 . Examining the terms in Eqs. B.2 and B.3, as well as the entries in Table 2 and Table 3 reveals 411 that the theories of Timoshenko, Novozhilov and Saada have largely similar governing 412 equations and boundary conditions. Thus, the theory of Timoshenko has been chosen as a 413 representative of this group. Similarly, the DMV theory has been taken as a representative of 414 the Morley-Koiter, Niordson and DMV group of theories given their similarity. In all 415 examples, results based on Abaqus models are provided for comparison. Four types of elements have been used in Abaqus models depending on the problem.

417 - S4R: A four-noded general-purpose (thin or thick) shell element, with reduced integration 418 and hourglass control.

419 - S8R: An eight-noded doubly curved thick shell element with reduced integration

- C3D8R: An eight-noded linearly interpolated brick element with reduced integration and hourglass control, and

- C3D20R: A twenty-node quadratically interpolated brick element with reduced integration. of $200 \mathrm{GPa}$ and a Poisson's ratio of $v=0.3$. 


\subsubsection{Example 1 - Pipe under Self-Weight}

426 A $4 \mathrm{~m}$ long fixed-free horizontal pipe with a radius $R=200 \mathrm{~mm}$ and a thickness $h=6 \mathrm{~mm}$ is subjected to its self-weight. The response is obtained based on the present theory, the Flugge,

428 Timoshenko, DMV theories, and the Abaqus shell model. In the Abaqus model, by refining 429 the mesh from $64 \times 200$ to $128 \times 400$ S4R elements, the changes in the predicted displacements were within $0.9 \%$, suggesting that convergence has been achieved. The static solution sought in the present problem is recovered by setting to zero the exciting frequency $\bar{\omega}$. Figure 5 provides a comparison of the longitudinal and radial displacements at the top generator of the pipe mid-surface. Nearly perfect agreement is observed among all solutions except for the DMV, which exhibits a significantly stiffer response as indicated by the lower displacements observed. A comparison of the predictions of the present and DMV theories shows a $53 \%$ difference (for a thickness $h=6 \mathrm{~mm}$ ). When the pipe thickness is reduced to $h=3 \mathrm{~mm}$, the difference between both solutions reduces to $22 \%$, drops further to $6.5 \%$ for $h=1.5 \mathrm{~mm}$, and becomes only $3 \%$ for $h=1 \mathrm{~mm}$. The fact that the DMV theory adopts the approximation $(1+z / R) \approx 1$, while the other theories do not enforce such an approximation, limits the usability of the DMV theory for very thin shells.

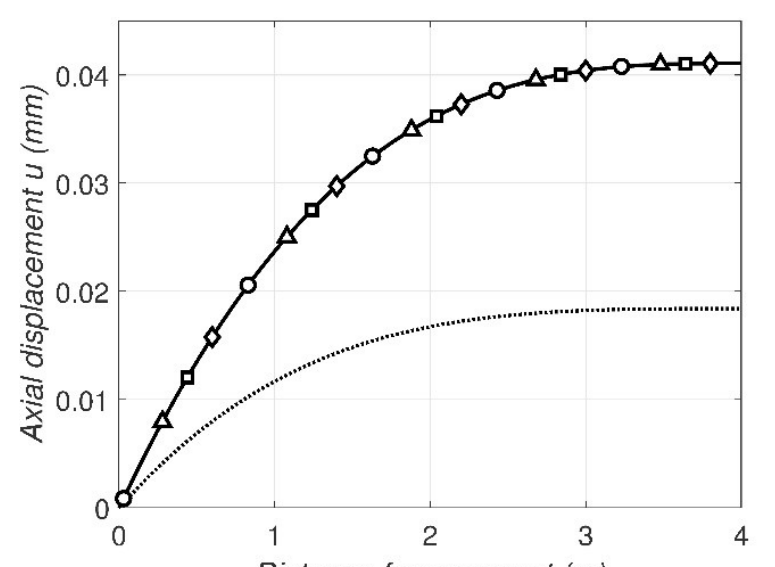

Distance from support ( $m$ )

Figure 5-Comparison of longitudinal and radial displacements at the top generator

\subsubsection{Example 2 - Fixed pipe under harmonic pressure}

444 Example 1 in [24] is revisited: A pipe with span length $L=5 \mathrm{~m}$, radius $R=25 \mathrm{~mm}$, thickness $445 h=5 \mathrm{~mm}$ is fixed at both ends and is subjected to wind load traction $t^{z}(x, s, t)=100 \cos (s / R) \cos (\bar{\omega} t) \mathrm{kN} / \mathrm{m}^{2}$, where $\bar{\omega}=200 \mathrm{rad} / \mathrm{s}$, simulating a vortex shedding 
447 phenomenon. Casting the applied traction in form of body forces applicable to the present 448 formulation yields $p^{z}=t^{z}(x, s, t) \operatorname{Dirac}(z-h / 2) N / m^{3}$. In addition to the present theory, 449 solutions are to be provided under the Flugge, Timoshenko, and DMV theories as well as 450 Abaqus FEA for comparisons. In the Abaqus model, by refining the mesh from $64 \times 200$ to $451128 \times 400$ S4R elements, the change in the predicted displacements was less than $0.05 \%$, 452 suggesting that convergence has been achieved by $64 \times 200$ mesh.

453

454

455

456

457

460

461

462

463

464

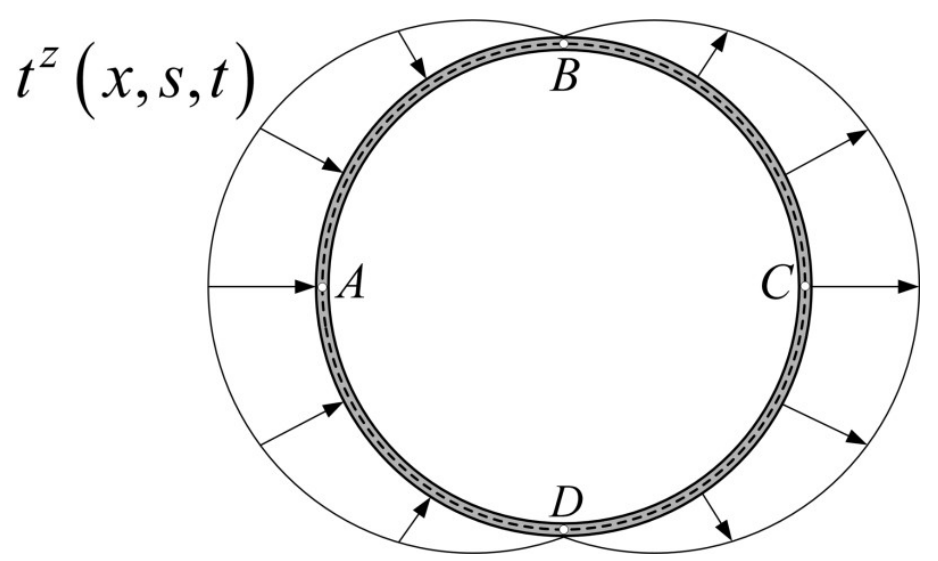

Figure 6 - Cross-sectional distribution of the wind load.

The maximum longitudinal and radial displacements at midsurface $(z=0)$ take place at the generators passing through points $A(s=0)$ and $C(s=\pi / 4)$ and the maximum circumferential midsurface displacement occurs at the generators passing through points $B$ ( $s=\pi / 8)$ and $D(s=3 \pi / 8)$, as shown in Figure 6. Table 4 gives the maximum displacements and their respective locations as provided by the present theory and percentage differences based on other solutions mentioned above. For the present problem, the predictions of the present theory are indistinguishable from those of the Flugge and Timoshenko theories, while the DMV solution slightly underpredicts the displacement by about $2.7 \%$. The Abaqus solution, also, is found to slightly underpredicting the displacement by less than $1 \%$. 
Table 4 - Comparing the displacement components at the location of their maximum

\begin{tabular}{|l|c|c|c|c|c|}
\hline & \multirow{2}{*}{ Present theory } & \multicolumn{4}{|c|}{ Difference from the present theory (\%) } \\
\cline { 3 - 6 } & & Flugge & Timoshenko & DMV & $\begin{array}{c}\text { Abaqus } \\
(64 \times 200 \text { S4R })\end{array}$ \\
\hline $\begin{array}{l}\bar{u}_{A}=-\bar{u}_{C} \text { at } x=0.21 L \\
-\bar{u}_{A}=\bar{u}_{C} \text { at } x=0.79 L\end{array}$ & $0.4410 \mathrm{~mm}$ & 0.00 & -0.01 & 2.73 & 0.91 \\
\hline $\bar{v}_{B}=-\bar{v}_{D}$ at $x=0.5 \mathrm{~L}$ & $3.753 \mathrm{~mm}$ & 0.00 & -0.01 & 2.72 & 0.92 \\
\hline $\bar{w}_{A}=-\bar{w}_{C}$ at $x=0.5 \mathrm{~L}$ & $-3.725 \mathrm{~mm}$ & 0.00 & -0.01 & 2.72 & 0.92 \\
\hline
\end{tabular}

466

467 The distribution of longitudinal stresses $\sigma_{x x}$ and transverse stresses $\sigma_{s s}$ for the generator 468 passing through point $A$ (Figure 6) are depicted in Figure 7 and Figure 8. Flugge's theory 469 predictions nearly coincide with those of the present solution, and the Timoshenko theory 470 predictions are in rather close agreement. The predictions of the DMV theory are 1-3\% below 471 those based on the other theories. Although, in the present example, all four theories predict 472 stresses in rather close agreement, this will not always be the case. For instance, if all degrees 473 of freedom at end $x=L$ are released (turning the beam into a cantilever), the stresses predicted 474 by the DMV are found to depart from those predicted by the Timoshenko, Flugge and present 475 theories. Figure 9 depicts the longitudinal stress $\sigma_{x x}$ along a generator passing through point $476 A$ (in Figure 6) under only $20 \%$ of the magnitude of $t^{z}(x, s, t)$ (to keep the maximum stresses 477 within the elastic range of the structural steel properties). The figure shows that the DMV 478 prediction significantly departs from those of the other theories. 


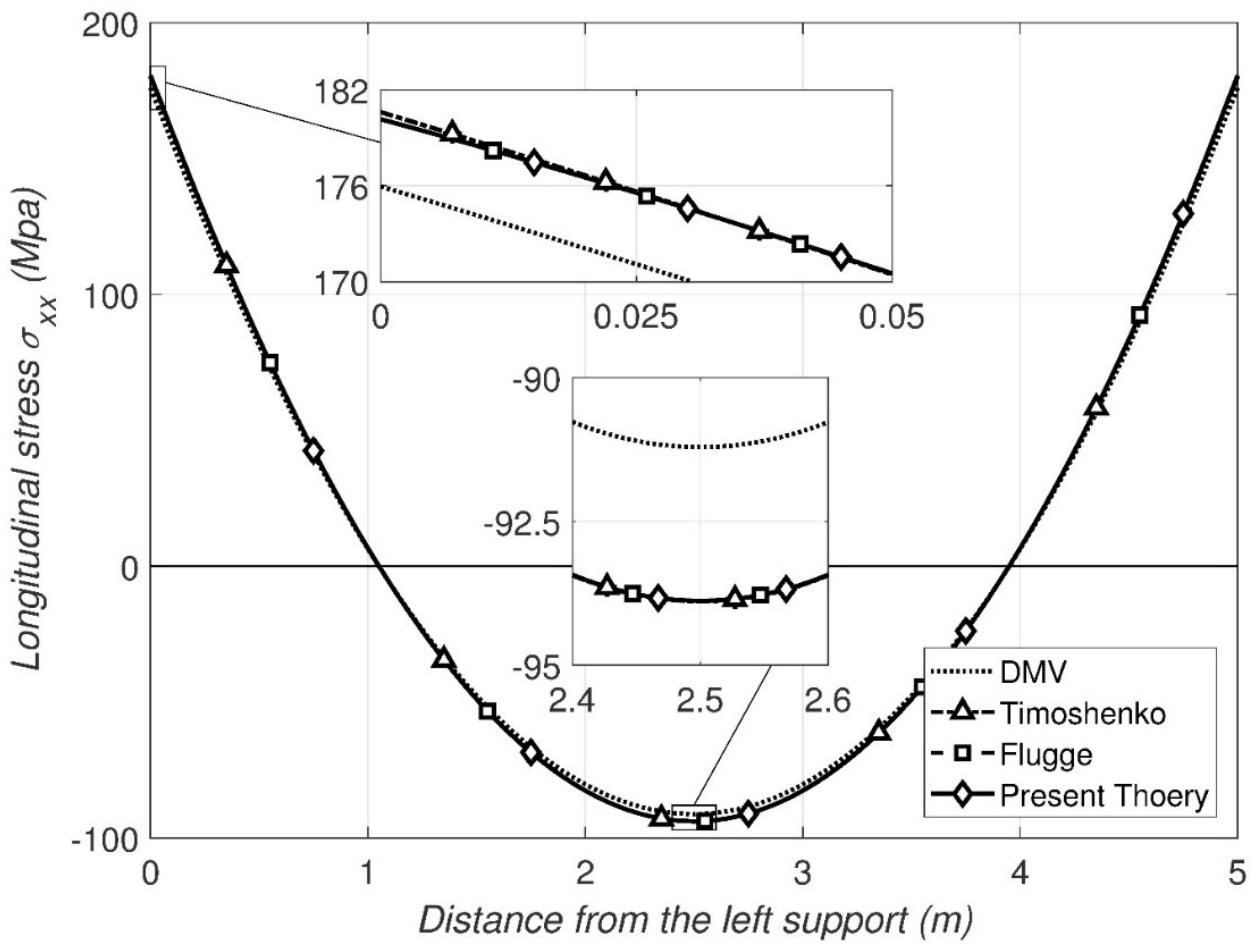

Figure 7 - Longitudinal stress $\left(\sigma_{x x}\right)$ along a generator passing through point $\mathbf{A}$

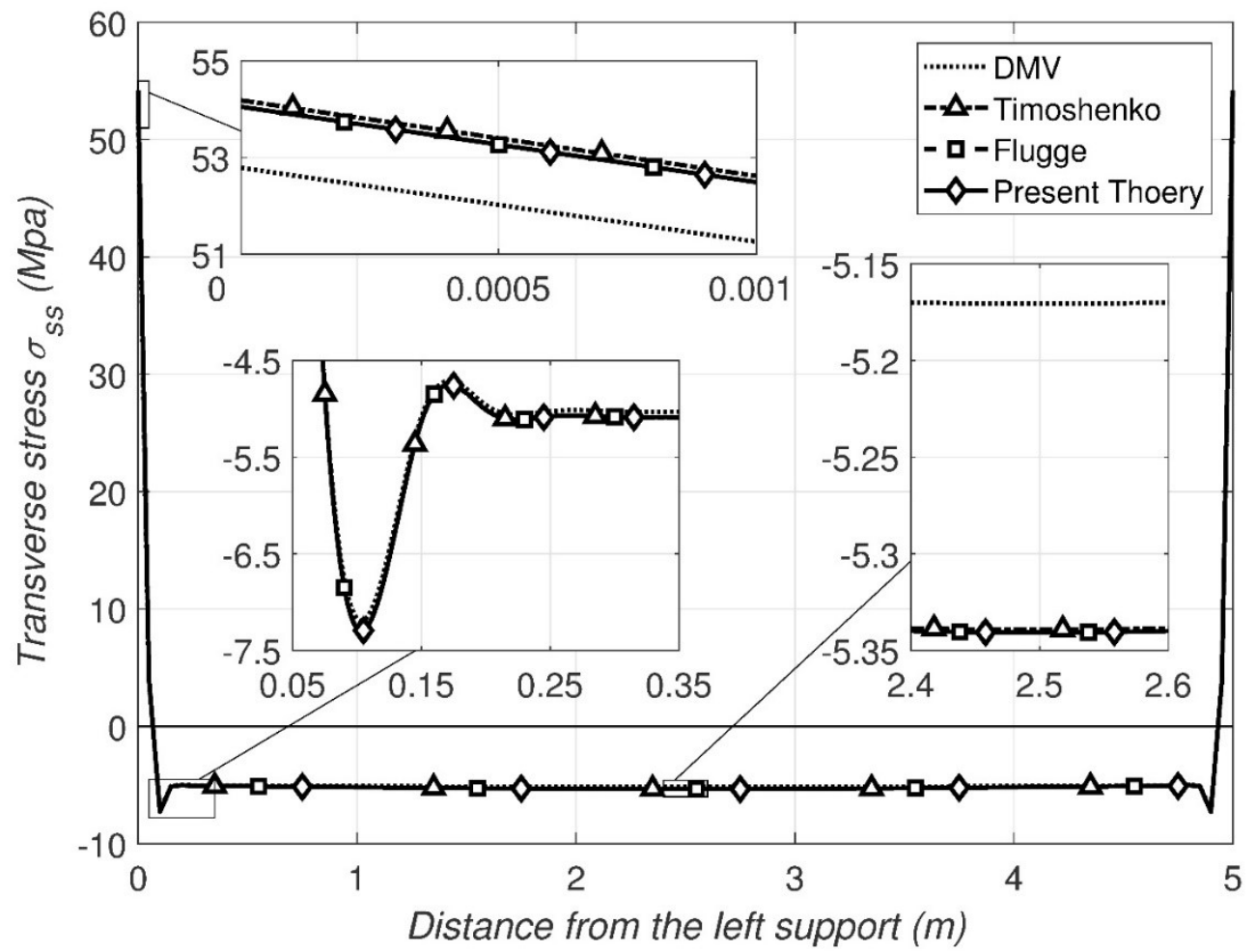

Figure 8 - Transverse stress $\left(\sigma_{s s}\right)$ along a generator passing through point $\mathbf{A}$ 


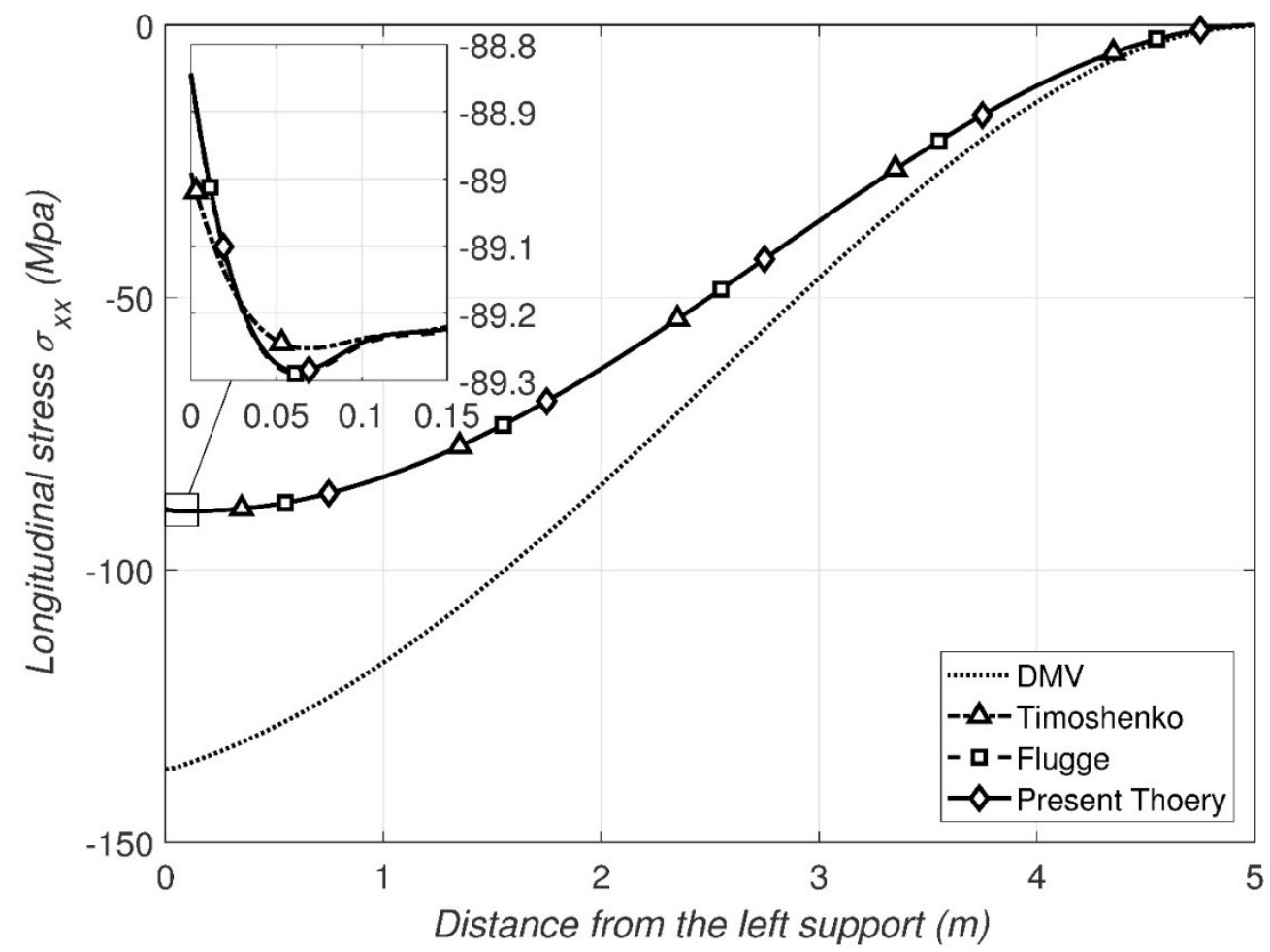

484

485

486

487

488

489

490

491

492

493

494

495

496

497

498

499

500

Figure 9 - Longitudinal stress $\left(\sigma_{x x}\right)$ along a generator passing through point $A$ for cantilever

The amplitudes of the radial displacements at Point $A$ as predicted by the present solution are compared to those predicted by the Abaqus shell model for wide range of angular frequencies (Figure 10). In Abaqus, by refining the mesh from $80 \times 255$ to $160 \times 510$ S4R elements, the change in the predicted displacements was less than $0.9 \%$ for frequencies ranging from 0 to 200 $\mathrm{rad} / \mathrm{s}$, suggesting that convergence has been achieved. The radial displacement amplitudes (as identified on the scale of the left vertical axis) based on both solutions are nearly in perfect agreement and are barely distinguishable within the scale of the figure. The loci of the peak amplitudes, indicative of the natural frequencies, are also in excellent agreement with Abaqus predictions. Also shown on the figure is the percentage difference of the radial displacement at point A between both solutions, as identified on the scale of the right vertical axis. Except near the natural frequencies where both solutions approach infinity, or where the amplitude approaches zero, the amplitude difference is within 3\%. The comparison indicates that the present theory predicts lower natural frequencies for most of the frequency range and higher amplitudes compared to Abaqus. 


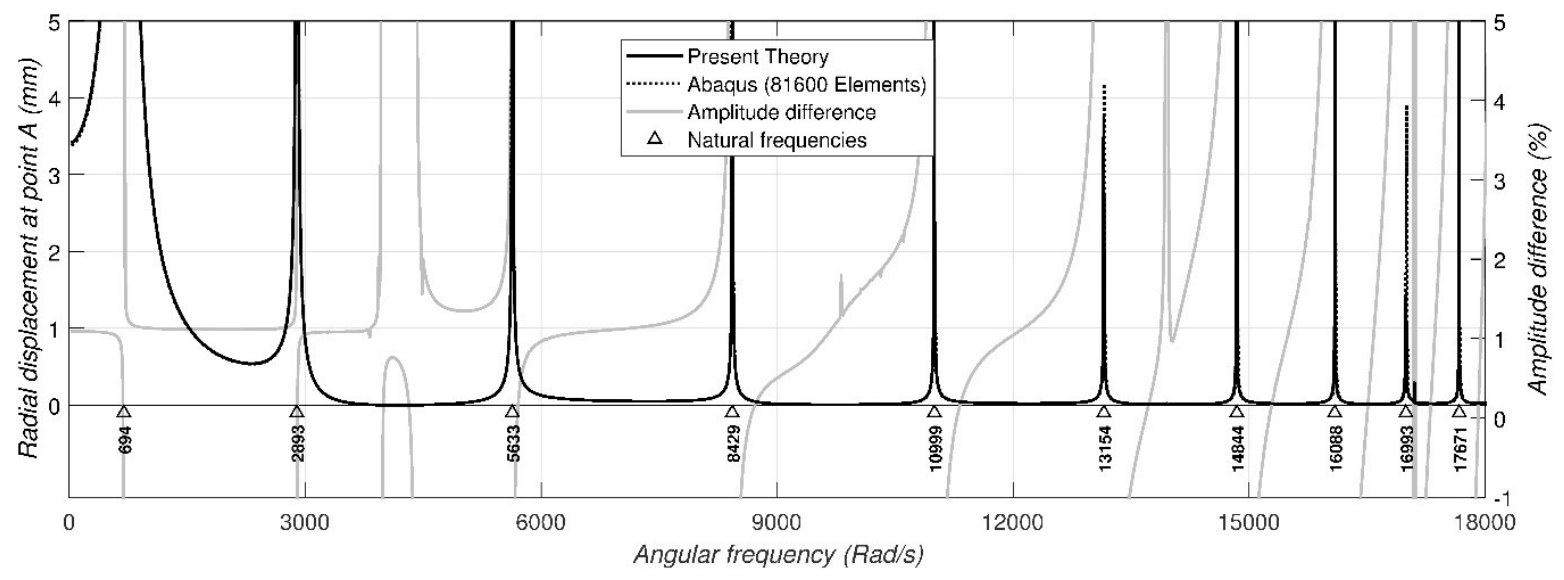

\subsubsection{Example 3 - Pipe under a Point Load}

505 The fixed pipe in Example 2 is set free at end $x=L$ and is subject to an inclined point load $506(1,1,-1) k N$ (Figure 11) along the $x, S$ and $z$ directions, acting at point $507 \quad P_{0}(x, s, z)=(0.7 L, 0,0)$.

508 509

510 511 512 513 514 515

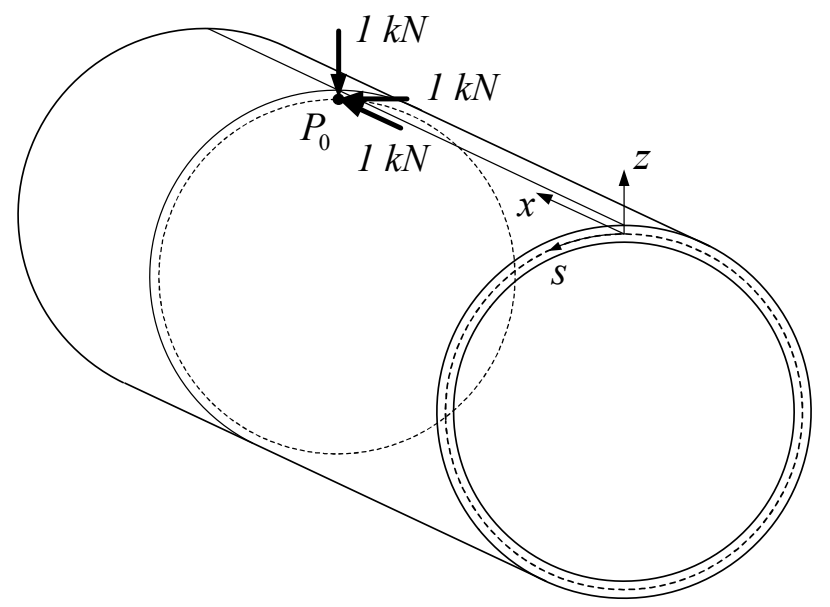

Figure 11 - Location, magnitude and direction of the point load components

The point loads can be expressed as body forces $\left(p^{x}, p^{s}, p^{z}\right)=\operatorname{Dirac}(x-0.7 L) \operatorname{Dirac}(s) \operatorname{Dirac}(z)(1,1,1) k N$ and expanded as double Fourier series in the $x$ and $z$ directions. To investigate the convergence of the solution, the number of Fourier modes taken is varied ( $N=K=7,10,15,20,25)$. Five Abaqus S4R shell models, with uniform meshes, were conducted for comparison $(16 \times 50,32 \times 100,64 \times 200,128 \times 400$ and $320 \times 640)$. The four coarse meshes are uniform while the most refined mesh $(320 \times 640$ 
516 ) is identical to the $128 \times 400$ mesh, with a refined rectangular patch of elements in the

517 neighborhood of the point load. The patch extends longitudinally from $x=0.6 L$ to $x=0.8 L$ 518 and from $s=-\pi R / 2$ to $s=\pi R / 2$ in the circumferential direction and consists of $256 \times 320$

519 elements (instead of $64 \times 80$ elements in the $128 \times 400$ elements model), and is intended to 520 capture localized deformations around the point load. In Abaqus, a mesh study indicated that 521 convergence was achieved for $128 \times 400$ S4R mesh. A finer $320 \times 640$ mesh resulted only in 522 a maximum difference of $0.23 \%$ in the displacements predicted.

523 The longitudinal, circumferential, and radial displacements at the generator at which the point 524 load is applied ( $s=z=0)$ are respectively depicted in Figure 12 to Figure 14. As the number 525 of Fourier terms increases in the present solution, and as the number of elements increase in 526 the Abaqus solution, both models are observed to approach one another, albeit in the limit, 527 both converged solutions do not exactly coincide. The rather minor differences observed arise 528 from the fact that an exact representation of the point load requires an infinite number of modes $529(N \rightarrow \infty \& K \rightarrow \infty)$, which is unachievable as the number of modes taken has to be 530 truncated. To confirm this hypothesis, an additional Abaqus solution is conducted (denoted as 531 A. $128 \times 640 N=K=7$ ). The applied point loads (originally modelled as nodal forces in the 532 shell model) were replaced by applied tractions that follow the exact distribution based on the 533 first seven Fourier modes, in a manner consistent with the load characterization in the present 534 solution (denoted as P. T. $N=K=7$ ). As expected, both solutions (P. T. $N=K=7$ ), and 535 (A. $128 \times 640 N=K=7$ ) predict nearly identical displacements.

536 Locally, the Abaqus shell solution exhibits minor displacement fluctuations within the vicinity 537 of the applied point load while the present solution predicts smoother displacement profiles. 538 Two additional Abaqus models were developed based on the S8R shell element and the 539 C3D20R brick element (both elements using quadratic interpolations as opposed to linear 540 interpolation in the S4R element). For the C3D20R, two layers of elements were taken across 541 the pipe thickness. Both models (not shown on Figure 12 to Figure 14 for clarity) were found 542 to exhibit less fluctuation within the vicinity of the point load than the S4R model, i.e., their 543 response is found closer to that of the present model. 

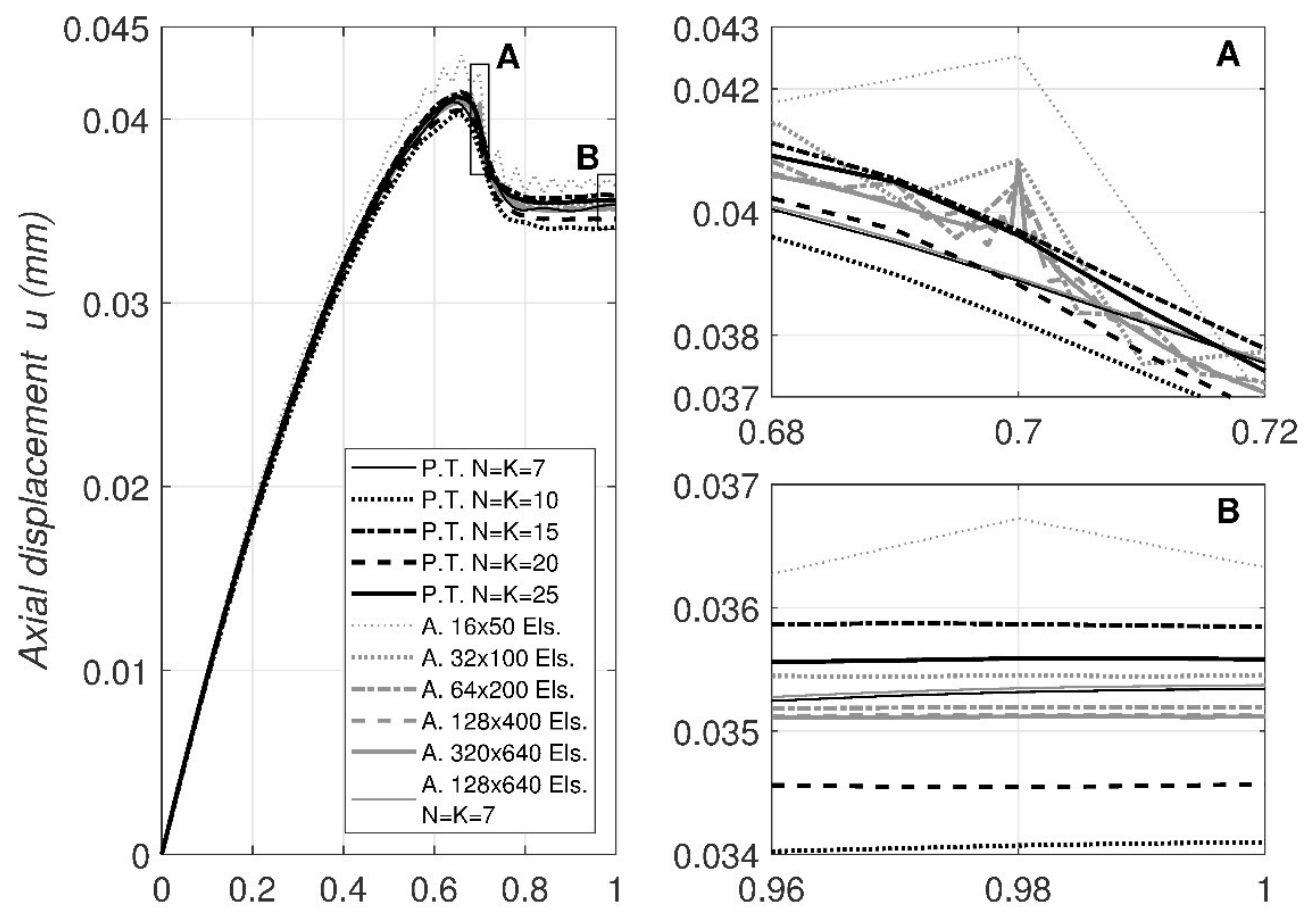

Figure 12 - Comparison of longitudinal displacements at top generator
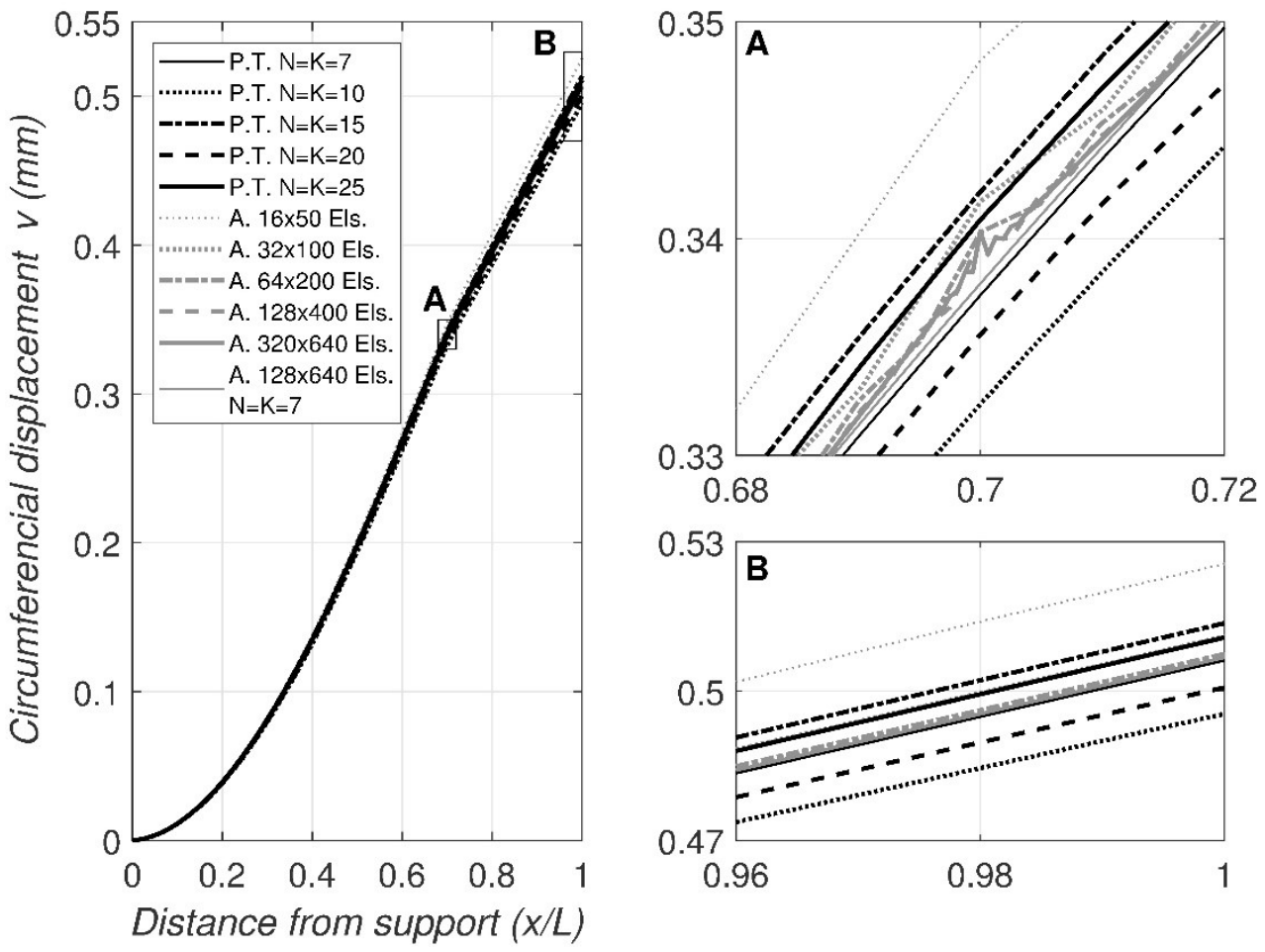

Figure 13 - Comparison of circumferential displacements at top generator 
551

552

553 To investigate the effect of span on the results, three spans are considered; $L=0.5 \mathrm{~m}$,

$554 L=2.5 \mathrm{~m}$ and $L=5 \mathrm{~m}$. The displacement components of the top generator $(s=z=0)$ versus

555 the normalized longitudinal coordinate $x / L$ are provided in Figure 15 to Figure 17. The 556 number of modes taken is $N=K=25$, which is beyond the number of modes required for 557 convergence. Abaqus S4R meshes were based 240 elements in the circumferencial direction 558 and 400 elements in longitudinal directions (a $240 \times 400$ mesh) for $\operatorname{span} L=0.5 \mathrm{~m}$, a $559128 \times 200$ mesh for $\operatorname{span} L=2.5 \mathrm{~m}$, and a $128 \times 640$ mesh for $\operatorname{span} L=5 \mathrm{~m})$.

560 As a general observation, all predictions are in very good agreement with the exception of the 561 DMV theory. In addition, the difference between the responses predicted by the DMV solution 562 and those of the other solutions tend to grow with the pipe span. In all cases, the DMV solution 563 underestimates the dispacements compared to other solutions.

564 The maximum difference between the present solution results and those based on Abaqus are 565 negligible. In general, the present theory predicts a slightly more flexible response compared 566 to that of the Abaqus model with fine meshes. Irrespective of the span, the present theory's 
567 predictions are in excellent agreement with Flugge's predictions, while the predictions of the 568 Timoshenko's theory tend to deviate slightly from those of the two theories for shorter spans.

569

570
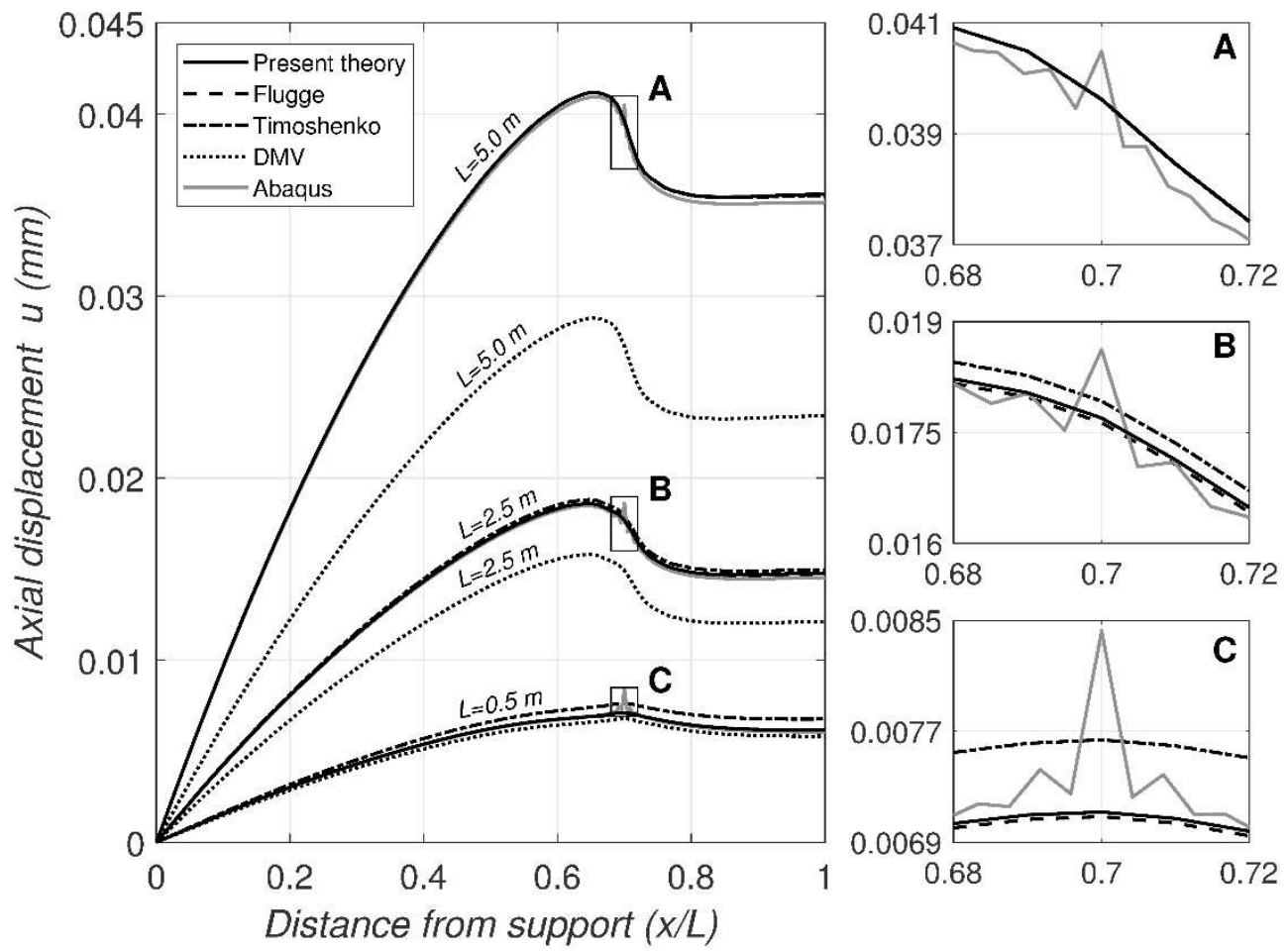

Figure 15 - Longitudinal displacement at the top generator
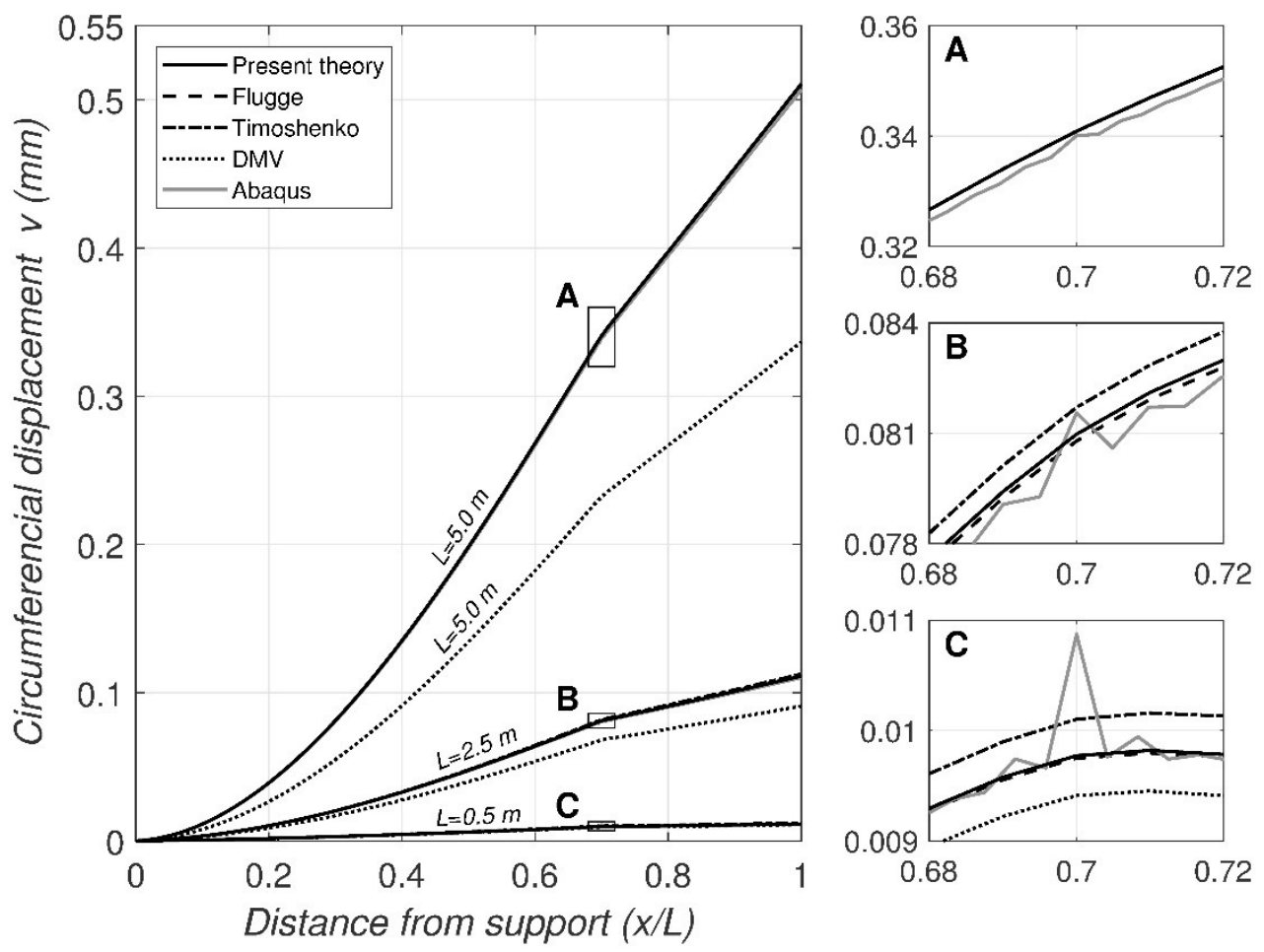

Figure 16 - Circumferential displacement at the top generator 
574

575

576

577

578

579

580

581

582

583

584

585

586

587 588
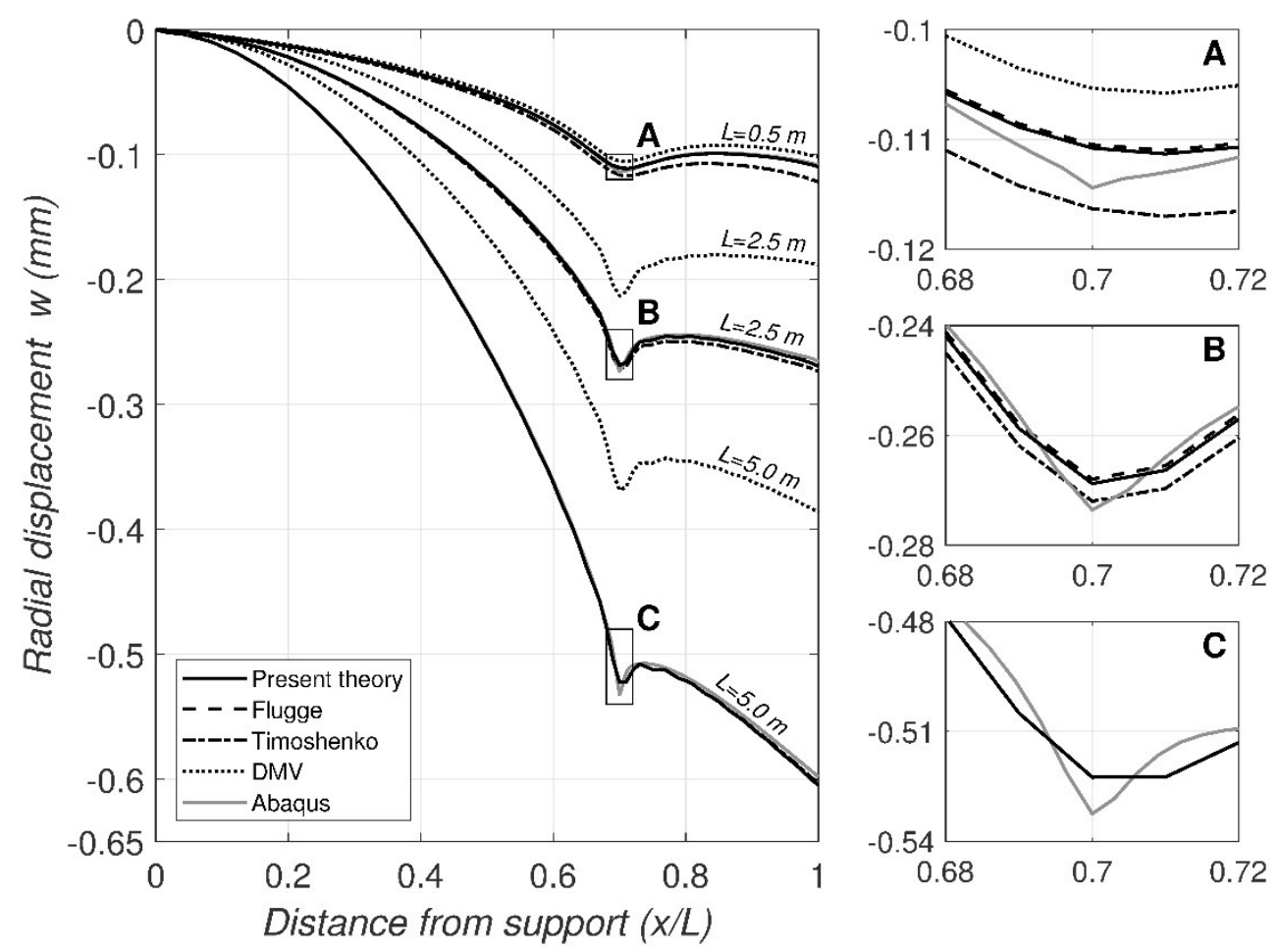

Figure 17 - Radial displacement at the top generator

To investigate the effect of thickness on various theories, the problem defined in the current example ( $L=5 \mathrm{~m}$ and $R=250 \mathrm{~mm}$, with the same point load) is solved again while varying the thickness to $h=5.0,12.5,25.0 \mathrm{~mm}$, which correspond to radius to thickness values of $R / h=50,20,10$, respectively). The problems are solved using two types of Abaqus models: one is based on $128 \times 400 \mathrm{~S} 4 \mathrm{R}$ Shell elements and the other is based on $4 \times 128 \times 400 \mathrm{C} 3 \mathrm{D} 8 \mathrm{R}$ Brick elements.

As shown in Figure 18 to Figure 20, predictions of the present theory, Flugge, and Timoshenko are in near perfect agreement, while Abaqus solutions provide an indistinguishable stiffer response. As the DMV results were far from the comparable range, they were not included in the plots. For the thicker pipe, the Timoshenko theory shows a negligibly more flexible response. In the proximity of the point load application, the finite element solutions exhibit localized oscillations, particularly for the brick element solution, which contrasts with the smoothness of the shell responses. 

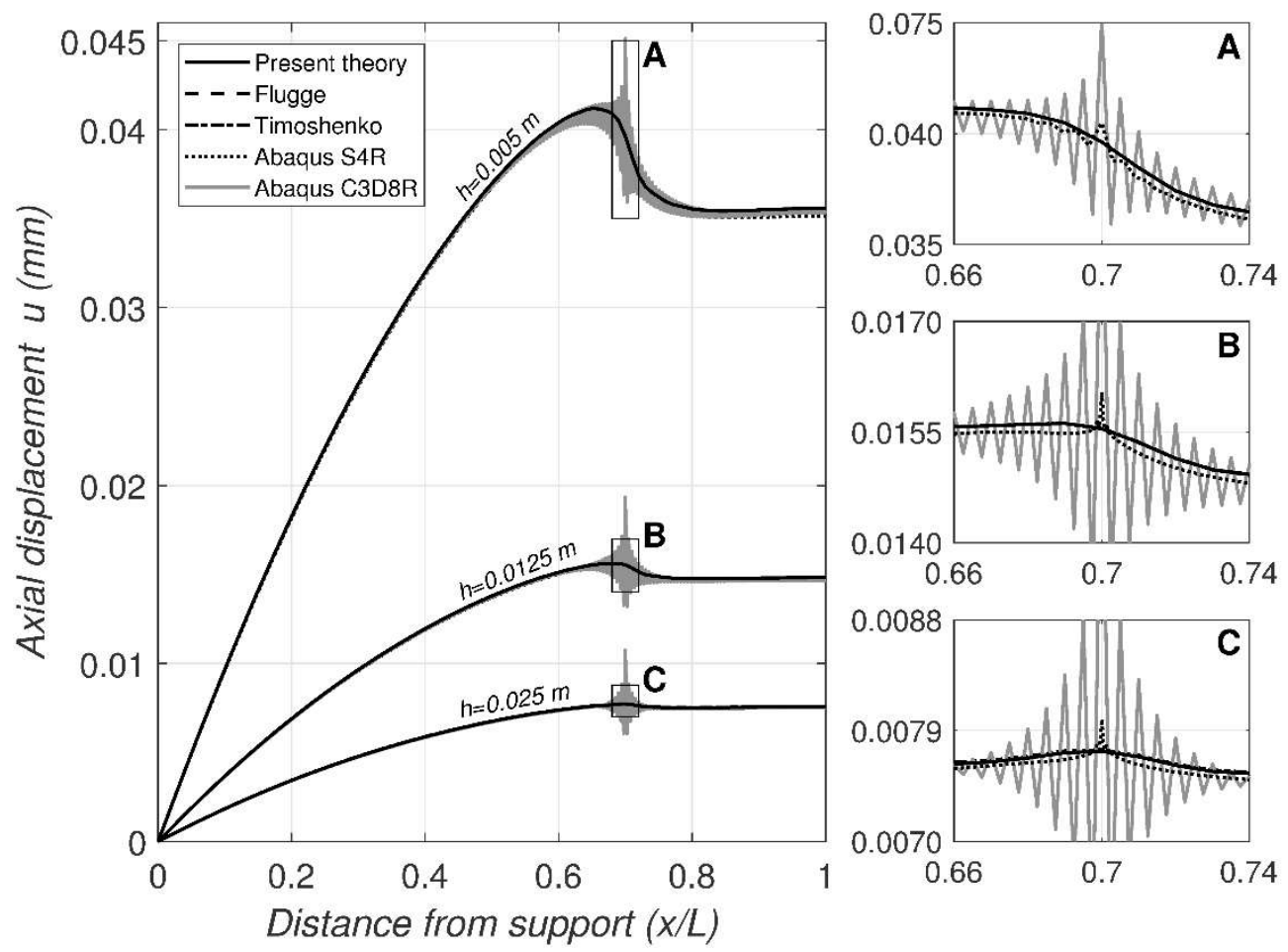

Figure 18 - Longitudinal displacement on the top generator for various theories and different Abaqus 591 elements 

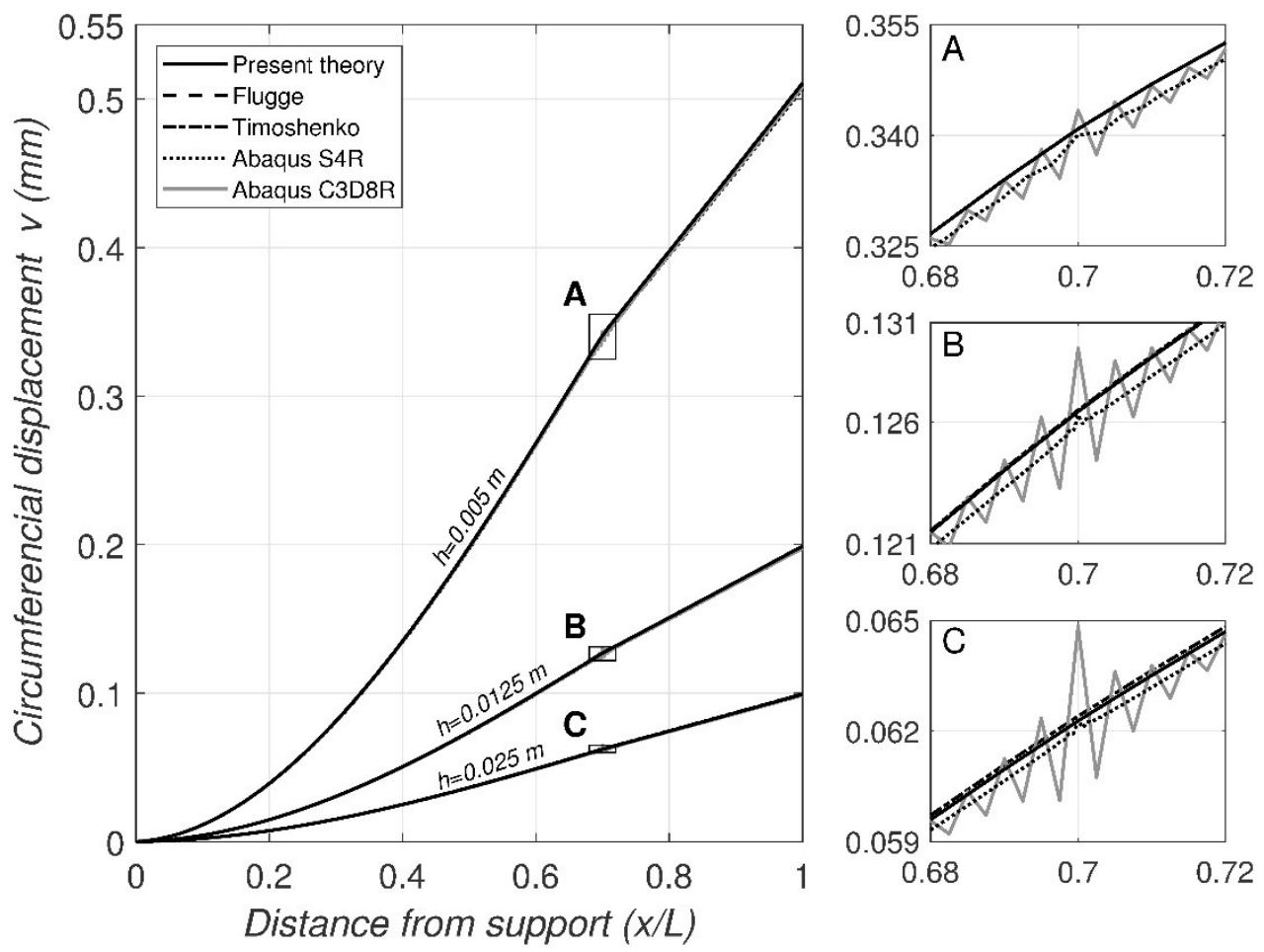

Figure 19 - Circumferential displacement on the top generator for various theories and different Abaqus 595 elements
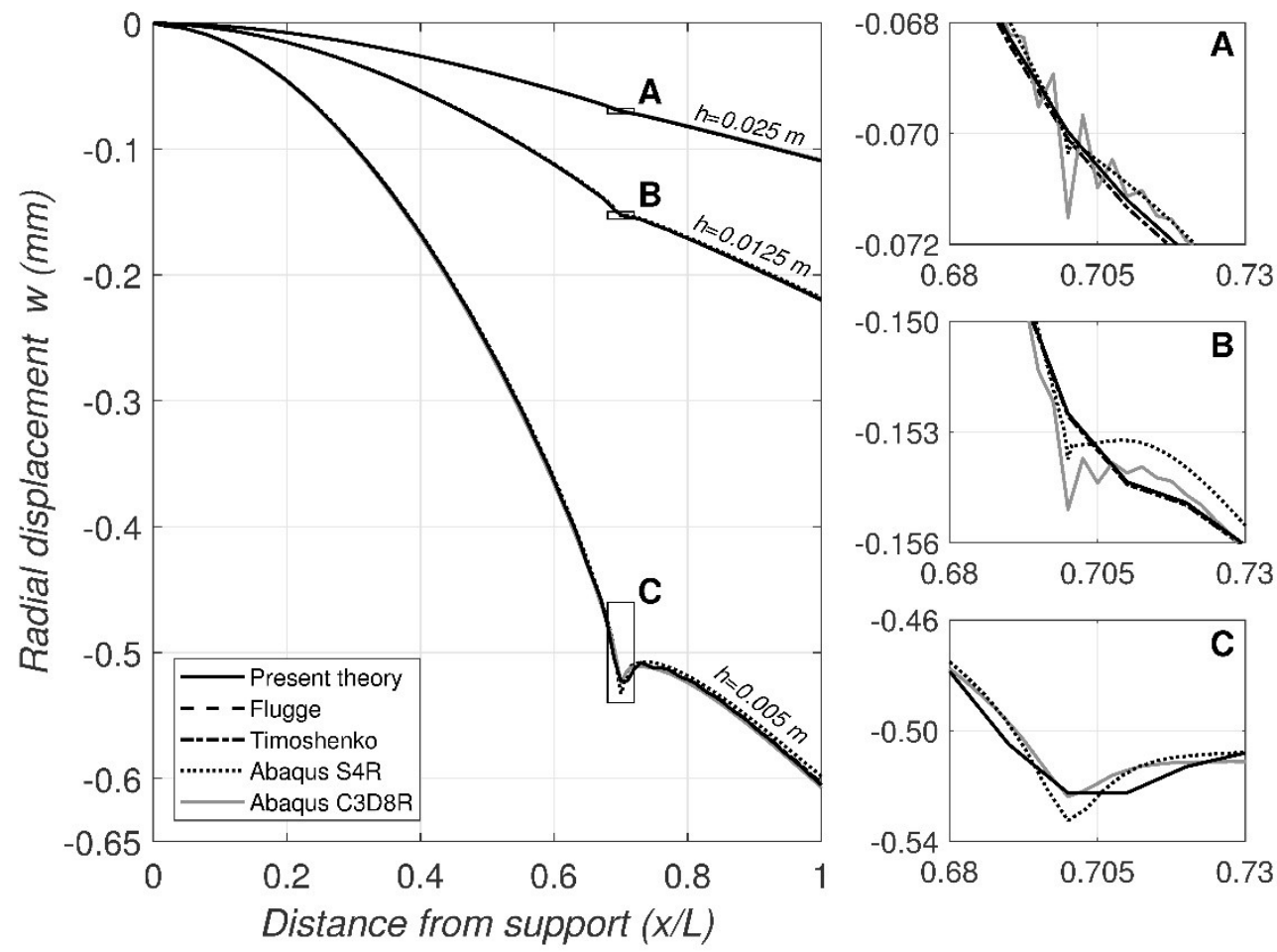

Figure 20 - Radial displacement on the top generator for various theories and different Abaqus elements 


\subsubsection{Example 4 - Comparisons against experimental results and analytical predictions}

601 The natural frequencies based on the present theory are compared to the experimental results

602 reported in [42]. The specimens tested were made of steel ( $E=200 G P a, \rho=7760 \mathrm{~kg} / \mathrm{m}^{3}$, $603 v=0.29$ ) with span $L=0.664 \mathrm{~m}$, radius $R=175 \mathrm{~mm}$, thickness $h=1.02 \mathrm{~mm}$. Figure 21

604 depicts excellent agreement between the predictions of the present theory and the experimental 605 results. Symbol $n$ denotes the circumferential wave number and symbol $\psi$ denotes the 606 longitudinal half-wave number and satisfies the relation $m=\psi \pi i / L$. The present theory tends 607 to predict slightly higher natural frequencies for higher circumferential modes. In all cases, 608 the maximum difference between the present theory predictions and experimental results is $609 \quad 1.5 \%$.

610

611

612 Additional comparisons are made against the analytical predictions in [43] which reported the 613 natural frequencies for a simply supported pipe made of rubber $(E=0.45 G P a, v=0.45$, $\left.614 \rho=1452 \mathrm{~kg} / \mathrm{m}^{3}\right)$, span $L=0.2 \mathrm{~m}$, radius $R=100 \mathrm{~mm}$, and thickness $h=2 \mathrm{~mm}$. Table 5 shows 615 that, for $\psi=1, \ldots, 8$ and $n=1, \ldots, 10$, the present theory is found to yield natural frequencies 616 of up to $1.36 \%$ lower than the values reported in [43]. 
617 Table 5 - Comparing natural frequencies based on the present theory with analytical results

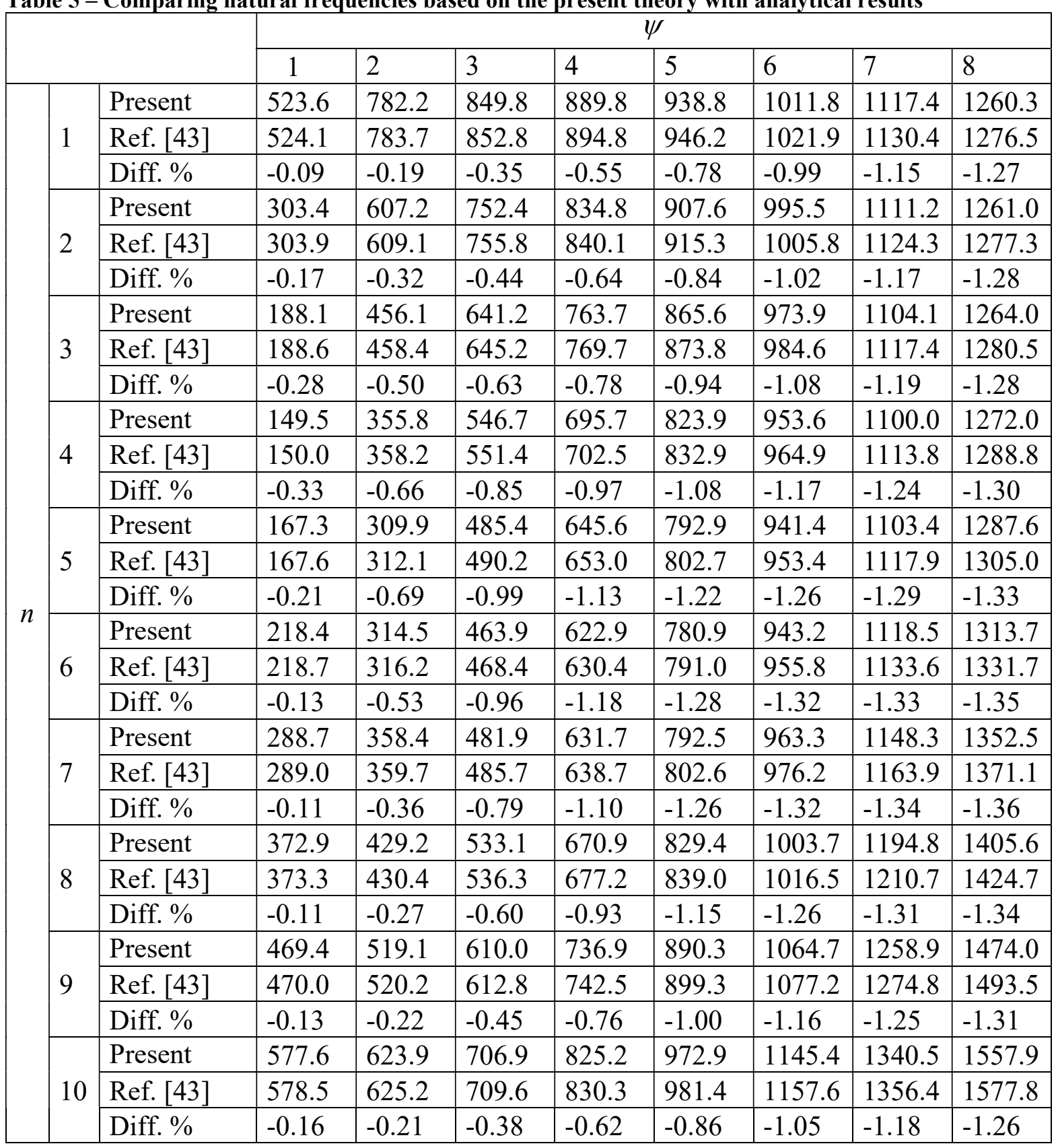

618 4. Summary and Conclusions

619 A variational expression is developed for the dynamic analysis of thin shells of general 620 geometries based on tensor calculus in conjunction with the Hamilton variational principle.

621 The solution is based on a minimal number of assumptions at the outset of the formulation 622 while avoiding non-essential approximations advocated in past theories. The variational 623 expression is then specialized to CCTS and used to formulate the governing equilibrium 624 equations and boundary conditions. The governing equations thus obtained are free from non625 essential approximations and can potentially serve as a benchmark to assess the accuracy of 
626 past circular cylindrical thin shell theories. The field equations derived are then compared to 627 those of Flugge, Timoshenko, Novozhilov, Morley-Koiter, Niordson, Saada and DMV. A 628 number of problems were numerically investigated using the present theory and comparisons 629 were made with the Flugge, Timoshenko, and DMV theories.

630 The main conclusions of the study are:

631 (1) The governing field equations of the Flugge and DMV theories were recovered as special 632 cases from the present theory by applying consistent approximations relating to the radius-to633 thickness ratio to the governing equations derived herein.

634 (2) Compared to the present treatment, the theories of Timoshenko, Novozhilov, Morley635 Koiter, Niordson, and Saada were found to involve inconsistent approximations. In some 636 cases, such inconsistencies lead to over-simplifications in the field equations, while in others, 637 they lead to the emergence of additional/unnecessary terms.

638 (3) The inconsistent simplifications made in the above theories do not necessarily correspond 639 to a significant reduction in their predictive power. Specifically, the inconsistencies involved 640 in Timoshenko's theory are of a small order and do not significantly influence its predictive 641 ability. For DMV, while the approximations made are of a consistent order, the order of 642 approximation implied happens to be too coarse to capture the response of pipes with moderate 643 thicknesses.

644 (4) Numerical predictions of the 3D and shell FEA solutions are in excellent agreement with 645 those of the present theory for a wide range of pipe dimensions $(2<L / R<20$ and $64610<R / h<50)$.

647 (5) The numerical predictions of the Flugge theory are found to be consistently in excellent 648 agreement with those of the present theory and Abaqus finite element solutions, and is thus 649 advocated as a more simplified CCTS theory, but yet as an accurate alternative to the present 650 theory.

651 (6) In contrast, DMV exhibits an overly stiff behaviour compared to the present and other 652 theories. In addition, it grossly overestimates the stresses in some cases. The theory is suitable 653 only for very thin pipes. 


\section{Acknowledgement}

655 The authors would like to express their deepest gratitude to the Baha'i Institute for Higher 656 Education (BIHE), Tehran, Iran for its encouragement and support to the first author. Also, 657 research funding from the Natural Science and Engineering Research Council of Canada 658 (NSERC) to the second author is gratefully acknowledged. 


\begin{tabular}{|c|c|}
\hline $\mathbf{a}_{i}$ & Covariant base vectors of the middle surface \\
\hline$a_{i j}$ & First fundamental tensor defined on the middle surface of a thin shell \\
\hline$a$ & Determinant of the first fundamental tensor $a_{i j}$ \\
\hline$b_{\alpha \beta}$ & Curvature tensor of the surfaces parallel and adjacent to the middle surface \\
\hline $\bar{b}_{\alpha \beta}$ & $\begin{array}{l}\text { Second fundamental tensor or curvature tensor defined on the middle } \\
\text { surface of a thin shell }\end{array}$ \\
\hline $\mathbf{a}^{i}$ & Contravariant base vectors of the middle surface \\
\hline$a^{i j}$ & $\begin{array}{l}\text { First fundamental tensor of the second kind defined on the middle surface } \\
\text { of a thin shell }\end{array}$ \\
\hline $\mathbf{g}_{i}$ & $\begin{array}{l}\text { Covariant base vectors of any surface parallel and adjacent to the middle } \\
\text { surface }\end{array}$ \\
\hline$g_{i j}$ & First fundamental tensor defined within the shell \\
\hline$g$ & Determinant of the first fundamental tensor $g_{i j}$ \\
\hline $\mathbf{g}^{i}$ & $\begin{array}{l}\text { Contravariant base vectors of any surface parallel and adjacent to the middle } \\
\text { surface }\end{array}$ \\
\hline$g^{i j}$ & First fundamental tensor of the second kind defined within the shell \\
\hline$p^{x}, p^{y}, p^{z}$ & $\begin{array}{l}\text { Physical components of the Contravariant body force components } F^{i} \text { as a } \\
\text { function of }(x, s, z, t)\end{array}$ \\
\hline $\bar{p}^{x}, \bar{p}^{y}, \bar{p}^{z}$ & $\begin{array}{l}\text { Physical components of the Contravariant body force components } F^{i} \text { as a } \\
\text { function only of }(x, s, z) \text {. }\end{array}$ \\
\hline $\mathbf{R}, \mathbf{r}$ & $\begin{array}{l}\text { Position vector of a point within the shell and on the middle surface, } \\
\text { respectively, before deformation. }\end{array}$ \\
\hline$\hat{\mathbf{R}}, \hat{\mathbf{r}}$ & $\begin{array}{l}\text { Position vector of a point within the shell and on the middle surface, } \\
\text { respectively, after deformation. }\end{array}$ \\
\hline $\mathbf{U}, \mathbf{u}$ & $\begin{array}{l}\text { Displacement of a point within the shell and on the middle surface, } \\
\text { respectively. }\end{array}$ \\
\hline$U_{i}, U^{i}$ & $\begin{array}{l}\text { Covariant and contravariant components of the displacement of a point on } \\
\text { a surface parallel and adjacent to the middle surface along axis } x^{i} \text {, } \\
\text { respectively. }\end{array}$ \\
\hline$u_{i}$ & $\begin{array}{l}\text { Covariant components of the displacement of a point on the middle surface } \\
\text { along axis } x^{i}\end{array}$ \\
\hline$u, v, w$ & $\begin{array}{l}\text { Components of the displacement of a point on the middle surface along axes } \\
x^{1}, x^{2} \text { and } x^{3} \text {, respectively }\end{array}$ \\
\hline$\chi^{i}$ & Coordinate system/Coordinates of the system \\
\hline$x^{i}$ & Coordinate system/Coordinates of the system \\
\hline$x, s, z$ & Coordinates of the system, equivalent to $x^{1}, x^{2}$ and $x^{3}$, respectively \\
\hline$\alpha, \alpha, \ldots$ & All Greek superscripts or subscripts range from 1 to 2 \\
\hline${ }_{i}{ }_{i}, \ldots$ & All Italic superscripts or subscripts range from 1 to 3 \\
\hline
\end{tabular}




\begin{tabular}{|l|l|}
\hline $\overrightarrow{\mathbf{i}}_{i}, \overrightarrow{\mathbf{j}}_{i}$ & $\begin{array}{l}\text { Covariant unit vectors along axis } x^{i} \text { in middle surface and adjacent } \\
\text { surfaces, respectively }\end{array}$ \\
\hline $\overrightarrow{\mathbf{i}}^{i}, \overrightarrow{\mathbf{j}}^{i}$ & $\begin{array}{l}\text { Contravariant unit vectors along axis } x^{i} \text { in middle surface and adjacent } \\
\text { surfaces, respectively }\end{array}$ \\
\hline$\Gamma_{i j}^{k}$ & Christoffel symbols in terms of $g_{i}$ and $g_{i j}$ \\
\hline $\bar{\Gamma}_{i j}^{k}$ & Christoffel symbols in terms of $a_{i}$ and $a_{i j}$ (defined for the middle surface) \\
\hline$\cdot$ & Inner product \\
\hline$\otimes$ & Cross product \\
\hline $\mid$ & Covariant derivative \\
\hline$\|$ & Covariant derivative in two dimensional spaces \\
\hline$T^{*}$ & Kinetic energy \\
\hline$V^{*}$ & Potential energy \\
\hline$V$ & Volume \\
\hline$W^{*}$ & External work \\
\hline$E_{i j}$ & Mechanical energy of the system \\
\hline$\eta_{i j}$ & Components of the strain tensor on the middle surface \\
\hline$\eta_{\alpha \beta}$ & Components of the strain tensor on surfaces adjacent to the middle surface \\
\hline$\tilde{\eta}_{\alpha \beta}$ & In-plane components of the strain tensor \\
\hline$\sigma^{i j}$ & Physical components of $\eta_{\alpha \beta}$ \\
\hline$\sigma^{\alpha \beta}$ & Stress tensor \\
\hline$\psi$ & In-plane components of the stress tensor $\sigma^{i j}$ \\
\hline$E^{i j l m}$ & Longitudinal half-wave number \\
\hline$E^{\alpha \beta \lambda \delta}$ & Generalized Hooke's Law \\
\hline$\cdots, i$ & In-plane components of the generalized Hooke's Law $E^{i j l m}$ \\
\hline$\delta_{\beta}^{\alpha}$ & Partial derivative with respect to $x^{i}$ \\
\hline$\mu_{\beta}^{\alpha}$ & Kronecker delta function \\
\hline$\lambda_{\alpha}^{\gamma}$ & $\begin{array}{l}\text { Mixed shifter tensor connecting the covariant base vectors of an arbitrary } \\
\text { point within the shell to corresponding base vectors of the middle surface. }\end{array}$ \\
\hline$\rho$ & Inverse of mixed shifter tensor $\mu_{\beta}^{\alpha}$ \\
\hline$E$ & Density of the mass \\
\hline$v$ & Modulus of elasticity \\
\hline$t$ & Poisson's ratio \\
\hline & time \\
\hline & \\
\hline & \\
\hline
\end{tabular}

661

662 


\section{References}

664 [1] S. Timoshenko, Theory of Plates and Shells. McGraw-Hill Book Company, New York, 665

666

667

668

669

670

671

672

673

674

675

676

677

678

679

680

681

682

683

684

685

686

687

688

689

690

691

692

693 1959.

[2] V.V. Novozhilov, Thin Shell Theory. P. Noordhoff Ltd. -Groningen-The Netherlands, 1964.

[3] W. T. Koiter, On the mathematical foundation of shell theory, Actes. Congres intern. Math. 3 (1970) 123-130.

[4] W. Flugge, Stresses in Shells. Springer-Verlag, New York Heidelberg Berlin, 1973.

[5] W. Flugge, Tensor Analysis and Continuum Mechanics, Springer-Verlag, New York Heidelberg Berlin, 1972.

[6] A. S. Saada, Elasticity: Theory and Applications, Pergamon Press Inc., New York, 1974.

[7] F. I. Niordson, Shell Theory. North-Holland Series In Applied Mathematics and Mechanics, 1985.

[8] R. W. Leonard, Nonlinear First Approximation Thin Shell and Membrane Theory. Ph. D. Dissertation, Virginia Polytechnic Institute, Blacksburg, Virginia, 1961.

[9] J. L. Sanders Jr., Nonlinear Theories for Thin Shells, Q. J. Appl. Math. 21 (1) (1963) 2136.

[10] W. T. Koiter, On the Nonlinear Theory of Thin Elastic Shells. Proceedings of the Koninklijke Nederlandse Akademie van Wetenschappen, Series B, 69 (1966) 1-54.

[11]W. T. Koiter, General Equations of Elastic Stability for Thin Shells. ProceedingsSymposium on the Theory of Shells, University of Houston, (1967), 187-227.

[12] B. Budiansky, Notes on Nonlinear Shell Theory, J. Appl. Mech. 35 (2) (1968) 393-401.

[13] A.W. Leissa, Vibration of Shells, Acoust. Soc. Am., 1993.

[14] A. Libai, C. W. Bert, A mixed variational principle and its application to the nonlinear bending problem of orthotropic tubes-I. Development of general theory and reduction to cylindrical shells, Int. J. Solids Struct. 31 (7) (1994) 1003-1018.

[15] A. -H. Muneeb, G. A. Birlik, Y. Mengi, A higher order dynamic theory for isotropic thermoelastic cylindrical shells: Part 1: Theory, J. Sound Vib. 179 (5) (1995) 817-826.

[16] S. V. Kolesnikov, A refined theory of the vibrations of a cylindrical shell based on an expansion in series of the normal displacement, J. Appl. Math. Mech. 60 (1) (1996) 113119. 
694

695

696

697

698

699

700

701

702

703

704

705

706

707

708

709

710

711

712

713

714

715

716

717

718

719

720

721

722

723

724

725

726

[17]F. I. Niordson, An asymptotic theory for circular cylindrical shells, Int. J. Solids Struct. 37 (13) (2000) 1817-1839.

[18] S. V. Ugrimov, Generalized theory of multilayer plates, Int. J. Solids Struct. 39 (4) (2002) 819-839.

[19] P. G. Ciarlet, L. Gratie, Another approach to linear shell theory and a new proof of Korn's inequality on a surface, Mathematical Problems in Mechanics, C. R. Acad. Sci. Paris, Ser. I 340 (2005).

[20] M. Birsan, On the theory of elastic shells made from a material with voids, Int. J. Solids Struct. 43 (2006) 3106-3123.

[21] H. Altenbach, V. A. Eremeyev, N. F. Morozov, Linear theory of shells taking into account surface stresses. Dokl. Phys. 54 (12) (2009) 531-535.

[22] K. Weicker, R. Salahifar, M. Mohareb, Shell analysis of thin-walled pipes. Part I - Field equations and solution, Int. J. Press. Vessels Pip. 87 (7) (2010) 402-413.

[23] K. Weicker, R. Salahifar, M. Mohareb, Shell analysis of thin-walled pipes. Part II - Finite element formulation, Int. J. Press. Vessels Pip. 87 (7) (2010) 414-423.

[24] R. Salahifar, M. Mohareb, Analysis of circular cylindrical shells under harmonic forces, Thin Walled Struct. 48 (7) (2010) 528-539.

[25] M. Amabili, J. N. Reddy, A new non-linear higher-order shear deformation theory for large-amplitude vibrations of laminated doubly curved shells, Int. J. Non Linear Mech. 45 (4) (2010) 409-418.

[26] V. N. Paimushin, A theory of thin shells with finite displacements and deformations based on a modified Kirchhoff-Love model, J. Appl. Math. Mech. 75 (5) (2011) 568-579.

[27] R. Salahifar, M. Mohareb, Finite element for cylindrical thin shells under harmonic forces, Finite Elem. Anal. Des. 52 (2012) 83-92.

[28] A. Favata, P. Podio-Guidugli, A new CNT-oriented shell theory, Eur. J. Mech. A. Solids 35 (2012) 75-96.

[29] C. Sansour, S. Skatulla, M. Hjiaj, A shell theory with scale effects and higher order gradients, Int. J. Solids Struct. 50 (2013) 2271-2280.

[30] J. Xuea, D. Yuana, F. Hanb, R. Liua, An extension of Karman-Donnell's theory for nonshallow, long cylindrical shells undergoing large deflection, Eur. J. Mech. A. Solids 37 (2013) 329-335.

[31] V. V. Zozulya, A high-order theory for functionally graded axially symmetric cylindrical shells, Arch. Appl. Mech. 83 (3) (2013) 331-343. 
727

728

729

730

731

732

733

734

735

736

737

738

739

740

741

742

743

744

745

746

747

748

749

750

751

752

753

754

755

[32]E Carrera, S. Brischetto, P. Nali, Plates and shells for smart structures: classical and advanced theories for modeling and analysis. John Wiley \& Sons, 2011.

[33] E. Carrera, M. Cinefra, M. Petrolo, E. Zappino, Finite element analysis of structures through unified formulation. John Wiley \& Sons, 2014.

[34] A. Cattabiani, H. Riou, A. Barbarulo, P. Ladevèze, B. Troclet, The Variational Theory of Complex Rays applied to the shallow shell theory, Comput. Struct. 158 (2015) 98-107.

[35] S. R. Chowdhurya, P. Roya, D. Roya, J. N. Reddy, A peridynamic theory for linear elastic shells, Int. J. Solids Struct. 84 (2016) 110-132.

[36] Ye. M. Zveryayev, A consistent theory of thin elastic shells, J. Appl. Math. Mech. 80 (2016) 409-420.

[37] J. Awrejcewicz, V. A. Krysko, A. A. Sopenko, M. V. Zhigalov, A. V. Kirichenko, A. V. Kryskoc, Mathematical modelling of physically/geometrically non-linear micro-shells with account of coupling of temperature and deformation fields, Chaos, Solitons Fractals 104 (2017) 635-654.

[38] Q. Wangab, D. Shaoc, B. Qind, A simple first-order shear deformation shell theory for vibration analysis of composite laminated open cylindrical shells with general boundary conditions, Compos. Struct. 184 (15) (2018) 211-232.

[39] R. Okhovat, A. Bostrom, Dynamic equations for an orthotropic cylindrical shell, Compos. Struct. 184 (2018) 1197-1203.

[40] Q. Wang, D. Shao, B. Qin, A simple first-order shear deformation shell theory for vibration analysis of composite laminated open cylindrical shells with general boundary conditions, Compos. Struct. 184 (2018) 211-232.

[41]R. Salahifar, Analysis of Pipeline Systems under Harmonic Forces, Ph. D. Thesis, Department of Civil Engineering, University of Ottawa, Ontario, Canada, 2011.

[42] M. Amabili, G. Dalpiaz, Breathing vibrations of a horizontal circular cylindrical tank shell, partially filled with liquid, J. Vib. Acoust. 117 (1995) 187-191.

[43] I. Senjanović, I. Ćatipović, N. Alujević, N. Vladimir, D. Čakmak, A finite strip for the vibration analysis of rotating circular cylindrical shells, Thin Walled Struct. 122 (2018) 158-172. 


$$
\begin{aligned}
& { }_{1}^{1} \bar{H}=-1 \quad, \quad{ }_{2}^{1} \bar{H}=-v \quad, \quad{ }_{3}^{1} \bar{H}=-v / R \quad, \quad{ }_{4}^{1} \bar{H}=h^{2} / 12 R \\
& { }_{1}^{2} \bar{H}=-(1-v) / 2 \quad, \quad{ }_{2}^{2} \bar{H}=-(1-v)\left(1+h^{2} / 4 R^{2}\right) / 2 \quad, \quad{ }_{3}^{2} \bar{H}=(1-v) h^{2} / 8 R \\
& { }_{1}^{3} \bar{H}=-h^{2} / 12 R \quad, \quad{ }_{2}^{3} \bar{H}=(1-v) R(\Phi-1) / 2 \quad, \quad{ }_{3}^{3} \bar{H}=-(3-v) h^{2} / 24 R \\
& { }_{4}^{3} \bar{H}=h^{2} / 12 \quad, \quad{ }_{5}^{3} \bar{H}=\left[(3-v) h^{2} / 24+(1-v) R^{2}(\Phi-1) / 2\right] \\
& { }_{6}^{3} \bar{H}=\rho\left(1-v^{2}\right) h^{2} / 12 R E \quad, \quad{ }_{7}^{3} \bar{H}=-\rho\left(1-v^{2}\right) h^{2} / 12 E \\
& { }_{8}^{3} \bar{H}=\left(1-v^{2}\right) / E h \\
& { }_{1}^{4} \bar{H}=h^{2} / 12 R, \quad \quad{ }_{2}^{4} \bar{H}=v h^{2} / 12 R \quad, \quad{ }_{3}^{4} \bar{H}=-h^{2} / 12 \\
& { }_{4}^{4} \bar{H}=-v h^{2} / 12 \\
& { }_{1}^{1} \bar{G}=1, \quad{ }_{2}^{1} \bar{G}=(1-v) \Phi / 2 \quad, \quad{ }_{3}^{1} \bar{G}=(1+v) / 2 \\
& { }_{4}^{1} \bar{G}=v / R \quad, \quad{ }_{5}^{1} \bar{G}=-h^{2} / 12 R \\
& { }_{6}^{1} \bar{G}=(1-v) R(\Phi-1) / 2 \quad, \quad{ }_{7}^{1} \bar{G}=-\rho\left(1-v^{2}\right) / E, \\
& { }_{8}^{1} \bar{G}=\rho\left(1-v^{2}\right) h^{2} / 12 R E \quad, \quad{ }_{9}^{1} \bar{G}=\left(1-v^{2}\right) / E h \\
& { }_{1}^{2} \bar{G}=(1+v) / 2 \quad, \quad{ }_{2}^{2} \bar{G}=(1-v)\left(1+h^{2} / 4 R^{2}\right) / 2 \quad, \quad{ }_{3}^{2} \bar{G}=1 \\
& { }_{4}^{2} \bar{G}=1 / R \quad, \quad{ }_{5}^{2} \bar{G}=-(3-v) h^{2} / 24 R \quad, \quad{ }_{a}^{2} \bar{G}=0 \\
& { }_{6}^{2} \bar{G}=-\rho\left(1-v^{2}\right)\left(1+h^{2} / 12 R^{2}\right) / E \quad, \quad{ }_{7}^{2} \bar{G}=\rho\left(1-v^{2}\right) h^{2} / 6 R E \quad, \\
& { }_{8}^{2} \bar{G}=\left(1-v^{2}\right) / E h \\
& { }_{1}^{3} \bar{G}=-v / R \quad, \quad{ }_{2}^{3} \bar{G}=-1 / R \quad, \quad{ }_{3}^{3} \bar{G}=-\Phi / R^{2} \\
& { }_{4}^{3} \bar{G}=-2(\Phi-1) \quad, \quad{ }_{5}^{3} \bar{G}=h^{2} / 12 R \\
& { }_{6}^{3} \bar{G}=-(1-v) R(\Phi-1) / 2 \quad, \quad{ }_{7}^{3} \bar{G}=(3-v) h^{2} / 24 R, \quad{ }_{b}^{3} \bar{G}=0 \\
& { }_{8}^{3} \bar{G}=-h^{2} / 12 \quad, \quad{ }_{9}^{3} \bar{G}=-\left[(3+v) h^{2} / 24+(1-v) R^{2}(\Phi-1) / 2\right] \\
& { }_{10}^{3} \bar{G}=-R^{2}(\Phi-1) \quad, \quad{ }_{11}^{3} \bar{G}=-\rho\left(1-v^{2}\right) / E \\
& { }_{12}^{3} \bar{G}=\rho\left(1-v^{2}\right) h^{2} / 12 E \quad, \quad{ }_{13}^{3} \bar{G}=\rho\left(1-v^{2}\right) h^{2} / 12 E \\
& { }_{14}^{3} \bar{G}=-\rho\left(1-v^{2}\right) h^{2} / 12 R E \quad, \quad{ }_{15}^{3} \bar{G}=-\rho\left(1-v^{2}\right) h^{2} / 6 R E \\
& { }_{18}^{3} \bar{G}=\left(1-v^{2}\right) / E h
\end{aligned}
$$

758 where $\Phi=\Phi(h / R)=(R / h) \ln [(2+h / R) /(2-h / R)]$ 
The terms $f^{x u}, f^{x w}, f^{s v}, f^{s w}, f^{z w}$ are related to the physical force components $p^{x}, p^{s}, p^{z}$ per 762 unit volume (of the contravariant external force components $F^{i}$, respectively) through the 763 integrals

$764\left\{\begin{array}{l}f^{x u} \\ f^{s v} \\ f^{z w} \\ f^{x w} \\ f^{s w}\end{array}\right\}=\int_{-\frac{h}{2}}^{+\frac{h}{2}}\left\{\begin{array}{c}p^{x}(1+z / R) \\ p^{s}(1+z / R)^{2} \\ p^{z}(1+z / R) \\ p^{x} z(1+z / R) \\ p^{s} z(1+z / R)\end{array}\right\} d z$

765 In Eq. 25, since $t_{1}$ and $t_{2}$ are arbitrary times and the variations $\delta u, \delta v, \delta w$ and $\delta w_{11}$ are 766 arbitrary within the domain $(0<x<L$ and $0<s<2 \pi R)$, the first and second integrals have 767 to vanish independently. The former integral yields eight boundary conditions,

768

$$
\begin{aligned}
& {\left[\left({ }_{1}^{1} \bar{H} u_{, 1}+{ }_{2}^{1} \bar{H} v_{, 2}+{ }_{3}^{1} \bar{H} w+{ }_{4}^{1} \bar{H} w_{, 11}\right) \delta u\right]_{0}^{L}=0} \\
& {\left[\left({ }_{1}^{2} \bar{H} u_{, 2}+{ }_{2}^{2} \bar{H} v_{, 1}+{ }_{3}^{2} \bar{H} w_{, 12}\right) \delta v\right]_{0}^{L}=0} \\
& \left.\left[\left({ }_{1}^{3} \bar{H} u_{, 11}+{ }_{2}^{3} \bar{H} u_{, 22}+{ }_{3}^{3} \bar{H} v_{, 12}+{ }_{4}^{3} \bar{H} w_{, 111}+{ }_{5}^{3} \bar{H} w_{, 122}+{ }_{6}^{3} \bar{H} \ddot{u}+{ }_{7}^{3} \bar{H} \ddot{w}_{, 1}+{ }_{8}^{3} \bar{H} f^{x w}\right) \delta w\right]\right]_{0}^{L}=0
\end{aligned}
$$

$$
\left[\left({ }_{1}^{4} \bar{H} u_{, 1}+{ }_{2}^{4} \bar{H} v_{, 2}+{ }_{3}^{4} \bar{H} w_{, 11}+{ }_{4}^{4} \bar{H} w_{, 22}\right) \delta w_{, 1}\right]_{0}^{L}=0
$$

$$
\begin{aligned}
& \left({ }_{1}^{1} \bar{G} u_{, 1}+{ }_{2}^{1} \bar{G} u_{, 22}+{ }_{3}^{1} \bar{G} v_{, 12}+{ }_{4}^{1} \bar{G} w_{, 1}+{ }_{5}^{1} \bar{G} w_{, 111}+{ }_{6}^{1} \bar{G} w_{, 122}+{ }_{7}^{1} \bar{G} \ddot{u}+{ }_{8}^{1} \bar{G} \ddot{w}_{, 1}+{ }_{9}^{1} \bar{G} f^{x u}\right)=0 \\
& \left({ }_{1}^{2} \bar{G} u_{, 12}+{ }_{2}^{2} \bar{G} v_{, 11}+{ }_{3}^{2} \bar{G} v_{, 22}+{ }_{4}^{2} \bar{G} w_{, 2}+{ }_{5}^{2} \bar{G} w_{, 112}+{ }_{a}^{2} \bar{G} w_{, 222}+{ }_{6}^{2} \bar{G} \ddot{v}+{ }_{7}^{2} \bar{G} \ddot{w}_{, 2}+{ }_{8}^{2} \bar{G} f^{s v}\right)=0 \\
& \left({ }_{1}^{3} \bar{G} u_{, 1}+{ }_{2}^{3} \bar{G} v_{, 2}+{ }_{3}^{3} \bar{G} w+{ }_{a}^{3} \bar{G} w_{, 11}+{ }_{4}^{3} \bar{G} w_{, 22}+{ }_{5}^{3} \bar{G} u_{, 111}+{ }_{6}^{3} \bar{G} u_{, 122}+{ }_{7}^{3} \bar{G} v_{, 112}+{ }_{b}^{3} \bar{G} w_{, 222}\right. \\
& +{ }_{8}^{3} \bar{G} w_{, 1111}+{ }_{9}^{3} \bar{G} w_{, 1122}+{ }_{10}^{3} \bar{G} w_{, 2222}+{ }_{11}^{3} \bar{G} \ddot{w}+{ }_{12}^{3} \bar{G} \ddot{w}_{, 11}+{ }_{13}^{3} \bar{G} \ddot{w}_{, 22}+{ }_{14}^{3} \bar{G} \ddot{u}_{, 1}+{ }_{15}^{3} \bar{G} \ddot{v}_{, 2} \\
& \left.+{ }_{16}^{3} \bar{G} f_{, 1}^{x w}+{ }_{17}^{3} \bar{G} f_{, 2}^{s w}+{ }_{18}^{3} \bar{G} f^{z w}\right)=0
\end{aligned}
$$

